\title{
The hypergiant HR 8752 evolving through the yellow evolutionary void ${ }^{\star} \star \star \star$
}

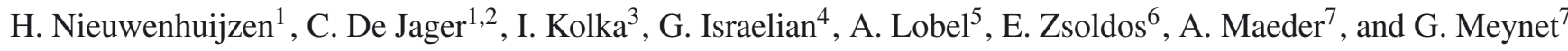 \\ 1 SRON Laboratory for Space Research, Sorbonnelaan 2, 3584 CA Utrecht, The Netherlands \\ e-mail: h.nieuwenhuijzen@sron.nl; h.nieuwenhuijzen@xs4all.nl \\ 2 NIOZ, Royal Netherlands Institute for Sea Research, PO Box 59, Den Burg, The Netherlands \\ e-mail: cdej@kpnplanet.nl \\ 3 Tartu Observatory, 61602 Tõravere, Estonia \\ e-mail: indrek@aai.ee \\ ${ }^{4}$ Instituto de Astrofisica de Canarias, via Lactea s/n, 38200 La Laguna, Tenerife, Spain \\ e-mail: gil@iac.es \\ 5 Royal Observatory of Belgium, Ringlaan 3, 1180 Brussels, Belgium \\ e-mail: alobel@sdf.lonestar.org; Alex.Lobel@oma.be \\ 6 Konkoly Observatory, PO Box 67, 1525 Budapest, Hungary \\ e-mail: zsoldos@konkoly.hu \\ 7 Observatoire de Genève, 1290 Sauverny, Switzerland \\ e-mail: [Andre.Maeder; Georges.Meynet]@unige.ch
}

Received 30 April 2011 / Accepted 13 March 2012

\begin{abstract}
Context. We study the time history of the yellow hypergiant HR 8752 based on high-resolution spectra (1973-2005), the observed MK spectral classification data, $B-V$ - and $V$-observations (1918-1996) and yet earlier $V$-observations (1840-1918).

Aims. Our local thermal equilibrium analysis of the spectra yields accurate values of the effective temperature $\left(T_{\text {eff }}\right)$, the acceleration of gravity $(g)$, and the turbulent velocity $\left(v_{\mathrm{t}}\right)$ for 26 spectra. The standard deviations average are $82 \mathrm{~K}$ for $T_{\text {eff }}, 0.23$ for $\log g$, and $1.1 \mathrm{~km} \mathrm{~s}^{-1}$ for $v_{\mathrm{t}}$.

Methods. A comparison of $B-V$ observations, MK spectral types, and $T_{\text {eff }}$-data yields $E(B-V)$, "intrinsic" $B-V$, $T_{\text {eff }}$, absorption $A$, and the bolometric correction $\mathrm{BC}$. With the additional information from simultaneous values of $B-V, V$, and an estimated value of $R$, the ratio of specific absorption to the interstellar absorption parameter $E(B-V)$, the "unreddened" bolometric magnitude $m_{\text {bol, }, 0}$ can be determined. With HIPPARCos distance measurements of HR 8752, the absolute bolometric magnitude $M_{\mathrm{bol}, 0}$ can be determined. Results. Over the period of our study, the value of $T_{\text {eff }}$ gradually increased during a number of downward excursions that were observable over the period of sufficient time coverage. These observations, together with those of the effective acceleration $g$ and the turbulent velocity $v_{\mathrm{t}}$, suggest that the star underwent a number of successive gas ejections. During each ejection, a pseudo photosphere was produced of increasingly smaller $g$ and higher $v_{\mathrm{t}}$ values. After the dispersion into space of the ejected shells and after the restructuring of the star's atmosphere, a hotter and more compact photosphere became visible. From the $B-V$ and $V$ observations, the basic stellar parameters, $T_{\text {eff }}, \log M / M_{\odot}, \log L / L_{\odot}$, and $\log R / R_{\odot}$ are determined for each of the observational points. The results show the variation in these basic stellar parameters over the past near-century.

Conclusions. We show that the atmospheric instability region in the HR-diagram that we baptize the yellow evolutionary void actually consists of two parts. We claim that the present observations show that HR 8752 is presently climbing out of the "first" instability region and that it is on its way to stability, but in the course of its future evolution it still has to go through the second potential unstable region.
\end{abstract}

Key words. stars: atmospheres - stars: evolution - supergiants - stars: mass-loss - stars: fundamental parameters stars: variables: S Doradus

\section{Introduction}

We summarize in this section the status of knowledge on HR 8752 and the evolution of hypergiants and succinctly review the aims of this research.

\footnotetext{
* Appendix $\mathrm{A}$ is available in electronic form at http: //www . aanda.org

$\star \star$ Tables A.x and B.x are available in electronic form at the CDS via anonymous ftp to

cdsarc.u-strasbg.fr (130.79.128.5) or via

http://cdsarc.u-strasbg.fr/viz-bin/qcat?]/A+A/546/A105, and at the external site http://www . aai . ee/HR 8752
}

\subsection{The yellow hypergiants; HR 8752 in the literature}

The star HR 8752 is a member of a group of half a dozen massive stars, with zero age main sequence (ZAMS) masses of $20-40$ or even more solar masses, the yellow hypergiants. Another wellstudied member is $\rho$ Cas (Lobel et al. 1992, 1994, 1998, 2003; Israelian et al. 1999; Gorlova et al. 2006). These are sites of heavy mass-loss, which is sometimes episodic. The individual stars undergo large variations in temperature. These variations are the visible atmospheric manifestations of changes that are only semi-coupled to the star, underlying its (pseudo-) photosphere. The evolutionary changes of the underlying star have a 
timescale that is much longer than the stability timescale of the photosphere. We associate these stars with the luminous blue variables (LBVs).

The extended literature on HR 8752 shows a large variability in the spectral classification, and colour magnitudes. An early review was written by De Jager (1980, p. 101-102). The historical light curve from 1840 to about 1980 is shown by Zsoldos (1986a), with a bibliography on what was known about the star at that time. The star HR 8752 is a variable with an amplitude of about $0.2 \mathrm{mag}$, and its light variation has a characteristic timescale of about one year. It has main-sequence B1 companion (Stickland \& Harmer 1978), and its circumstellar envelope emits thermal radio emission (Smolinski et al. 1977; Higgs et al. 1978). An excellent spectral analysis of the star at one point in its changing history was published by Luck (1975). He found an effective temperature of $4000 \mathrm{~K}$, a $\log g$ value of about -2 , and a metal abundance that seems to have Solar-System values. A self-consistent spectrum synthesis analysis (Fry \& Aller 1975) suggests that HR 8752 has solar-type abundances. No evidence has been found of extended structures (Schuster et al. 2003) for the yellow hypergiants $\rho$ Cas, HR 8752, HR 5171a, and $\mu$ Cep within 0 .' $^{\prime} 1$ of the stars and, down to the Hubble Wide-Field Camera (WFPC2) noise level, at about $2^{\prime \prime}$. The satellite HIPPARCOS (with identification number HIP115361) has obtained colour-magnitude data that show short timescale variability (Nieuwenhuijzen \& De Jager 2000). Its Tycho photometric data is given in the specific Tycho colour system that has yet to be translated to the Johnson system for comparison purposes.

\subsection{The evolution of hypergiant stars; the yellow evolutionary void}

The yellow hypergiants have absolute magnitudes $M_{\text {bol }}$

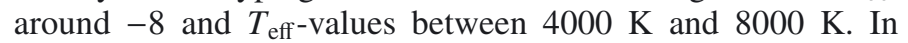
the HR diagram, they are situated at the low-temperature edge of an area that is virtually void of stars (Humphreys 1978; for LMC and other galaxies cf. also Humphreys \& Davidson 1979). A study of the void revealed that the few stars that are situated inside the void are young redward-evolving objects (Nieuwenhuijzen \& De Jager 2000). No (old) blueward evolving stars were found inside the void. It is generally assumed that the void is an area where blueward-evolving stars are unstable (De Jager \& Nieuwenhuijzen 1995; De Jager et al. 2001). This area of the Hertzsprung-Russell diagram (HR-diagram) was baptized the yellow evolutionary void, or yellow void in brief. Later in this paper, we show that the void actually contains two limited regions: $\log T_{\text {eff }} \approx 3.8-3.95$ where the ionization of $\mathrm{H}$ might give rise to a low stability of the atmosphere, and $\log T_{\text {eff }} \approx 4.05-4.1$ where the ionization of He plays a part. These separate regions lie within the general yellow void.

The position of some yellow hypergiants and the position of HR 8752 in the HR-diagram are given in Fig. 1, together with evolutionary tracks (Meynet et al. 1994) for ZAMS masses of 25 and 40 solar masses, respectively. The redwardevolving tracks are labelled "track 3" and the blueward-evolving "track 4". These tracks are also indicated in the figure. The reason for choosing these models is that we position HR 8752 (Nieuwenhuijzen \& De Jager 2000) somewhere between the models of a 25- and a 40-ZAMS solar-mass star (as given by the models of Meynet et al. 1994).

We call the transition region between these tracks the "mirror" point, this being a region between tracks 3 and 4, in the time after the main-sequence and the transition to the red
HR 8752 and evolutionary tracks (Meynet et al., 1994)

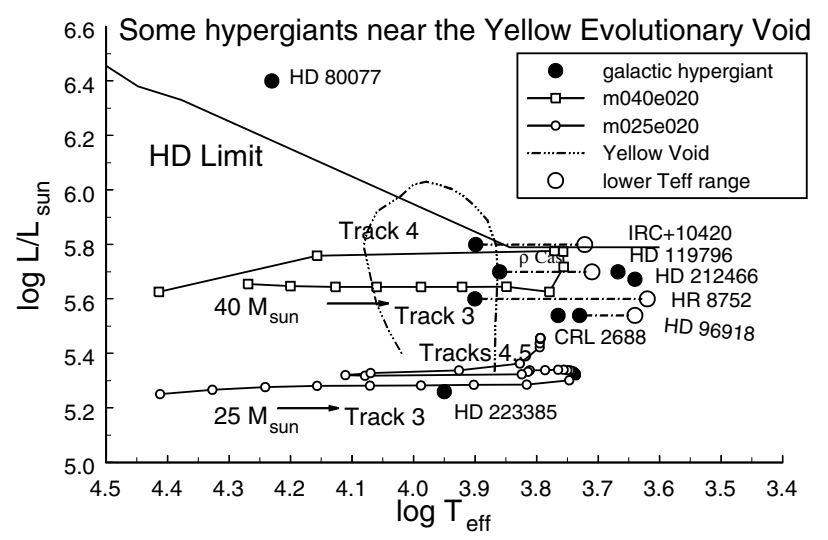

Fig. 1. Evolutionary tracks for 25 and 40 solar masses from Meynet et al. (1994), models with two times the mass-loss rate of De Jager et al. (1988). Some Galactic hypergiants including HR 8752 are indicated with their positions in the HR-diagram, some of them with observed changes of temperature. The Humphreys-Davidson limit and the approximate position of the yellow evolutionary void are indicated. For each model, evolutionary track 3 starts at the higher temperatures and evolves redward until the "mirror point" and then returns to higher temperatures as track 4. For the ZAMS 40 solar-mass model, track 4 goes to high temperatures, while for the ZAMS 25 solar-mass model track 4 goes to an inversion (a secondary "mirror" point) and thereupon goes redward as track 5 .

supergiant phase. The effective temperature $T_{\text {eff }}$ hardly changes during this time and large mass-losses occur.

An example of the timescales involved: for a model of ZAMS mass of 25 solar masses (Meynet et al. 1994) with a luminosity $\log \left(L / L_{\odot}\right)=5.3$, the time for evolution through the area of the yellow void corresponds to 10000 or more years, with an increase in temperature of about $1000 \mathrm{~K}$. While details differ for other models, it is clear that, because of the short time span involved, only few stars can be found inside the yellow evolutionary void.

There may also be another reason for the existence of this area of "avoidance". In an earlier paper by two of us (Nieuwenhuijzen \& De Jager 1995), we developed a code to

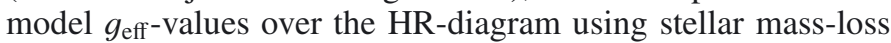
data (from De Jager et al. 1988) and mass data from stellar evolutionary models (Maeder \& Meynet 1988). For the mass, we differentiated the various evolutionary phases by using the above-mentioned tracks in a specific way (Nieuwenhuijzen \& De Jager 1995). In the course of the above calculations, it appeared that massive stars of ZAMS masses between 20 and about 60 solar masses are able to cross the HR diagram in their redward evolution (track 3) unimpededly, but on their return to the blue (track 4, cf. Fig. 1 where tracks 3 and 4 are indicated for a $40 M_{\odot}$ and a $25 M_{\odot}$ model) they have lost so much of their mass that their photospheres obtain effective accelerations close to zero and the mean weighted value of the thermodynamic coefficient $\left\langle\Gamma_{1}\right\rangle$ (defined in Eq. (1), Sect. 4.1) comes in a range where $\left\langle\Gamma_{1}\right\rangle<4 / 3$ over an appreciable part of the atmosphere. Such a star may then become unstable (not necessarily dynamically, but generally, cf. Maeder 2009, Sect. 1.3.2). In the outer layers of the hypergiant both below and above the photosphere all hydrodynamic processes are strongly nonadiabatic owing to the large radiation losses. In particular, instead of dynamical instability one may expect, for example, the strange mode oscillations. 
For certain areas in the HR-diagram, that coincide with the position of the void, no stable solution could be derived with our code, indicating the (possible) presence of a density inversion. This explanation seems reasonable owing to the development of the code that was defined for a monotonically decreasing atmospheric density, with scaleheights and the concept of $g_{\text {eff }}$ (loc. cit. Eqs. (6)-(9)). Although the existence of a density inversion does not directly lead to instability (cf. Maeder 2009, Sect. 5.5.2, and also Maeder 1989), it might begin to explain the existence of (massive) shells as we discuss in Sect. 6. The existence of this phenomenon coincides with low values of $\Gamma_{1}$, which is the driving parameter in this part of our research. The low values of $\Gamma_{1}$ in stars such as HR 8752 result from high radiation pressure and partial ionization effects. In Sect. 4, we discuss this parameter and its values over the HR-diagram for stellar models traversing the void.

\subsection{Aims of the present research}

During the past two decades, we have collected a large number of high-resolution spectra of HR 8752 to study the details of its evolution. It is gratifying to note that this initiative was successful, because the study of these spectra allowed us, for the first time, to investigate the details of the evolution of a hypergiant star during a crucial phase of its evolution. Of particular interest are the various episodes of mass-loss and the recovery afterwards. That part of the investigation is completed by the inclusion of occasionally obtained spectra at earlier epochs.

By analysing data on colours and magnitudes obtained in the past century, the timeline of this research is extended, albeit not with the accuracy and details of the spectral investigations.

In the following sections of this paper, we first discuss the high-resolution spectra. Thereafter we deal with the colours and magnitudes. After that we consider the consequences of this investigation for our understanding of the evolution of this particular hypergiant and the possible consequences for the evolution in general of very massive stars.

\section{Spectral line investigations and their results}

We now describe the analysis of our spectra. The reason for starting this systematic series of observations was that an earlier study (Nieuwenhuijzen \& De Jager 2000) of the time history of this star had shown the occurrence of occasional mass ejections associated with lower $T_{\text {eff }}$ values, after which $T_{\text {eff }}$ increased gradually until another episode of mass-loss again led to another drop of $T_{\text {eff }}$. This zigzag behaviour appeared to occur for $T_{\text {eff }}$ values in the range from approximately $5000 \mathrm{~K}$ to around $7500 \mathrm{~K}$, which was reached in the year 2000. After this time, $T_{\text {eff }}$ increased further to $\approx 8000 \mathrm{~K}$. It was considered interesting to see whether a new period of mass ejection would occur, and which values of the physical parameters would be essential for "triggering" the ejection process. Another question was whether the star would asymptotically approach the high temperature end of the void at around $T_{\mathrm{eff}} \approx 9000-10000 \mathrm{~K}$.

We therefore worked on a semi-regular basis to obtain new high-resolution spectra of HR 8752. Observations listed as obs5 to obs26 were made at the La Palma and Tartu Observatories in the period from 2000/7 to 2005/10. Table 1 lists observation dates and observers. We included four earlier published spectra (analysed the same way) from Nieuwenhuijzen \& De Jager (2000) to extend the baseline and for comparison purposes. These are mentioned as obs1-obs4 in the above table.

To determine the time variation in the atmospheric parameters $T_{\text {eff }}$ (effective temperature), $\log g_{\text {eff }}$ (effective acceleration),
Table 1. Observations, dates, and observers.

\begin{tabular}{lccc}
\hline \hline Observations & Date & JD-2 400 000 & Observer \\
\hline obs1 & $1973.08 .13-17$ & 41911.3 & Luck \\
obs2 & $1978.08 .-$ & 43737.3 & Smolinski \\
obs3 & $1984.07 .-$ & 45898.3 & Piters \\
obs4 & 1995.04 .18 & 49824.3 & Rutten \\
obs5 & 2000.06 .06 & 51702.5 & Israelian \\
obs6 & 2000.10 .06 & 51824.4 & Kolka \\
obs7 & 2000.11 .27 & 51876.4 & Kolka \\
obs8 & 2001.01 .12 & 51922.3 & Kolka \\
obs9 & 2001.02 .16 & 51957.3 & Kolka \\
obs10 & 2001.03 .30 & 51999.4 & Kolka \\
obs11 & 2001.09 .25 & 52178 & Kolka \\
obs12 & 2001.10 .01 & 52184 & Israelian \\
obs13 & 2001.11 .16 & 52230.4 & Kolka \\
obs14 & 2002.03 .25 & 52359.6 & Kolka \\
obs15 & 2002.08 .21 & 52508.5 & Kolka \\
obs16 & 2002.12 .14 & 52623.4 & Kolka \\
obs17 & 2003.02 .19 & 52690.3 & Kolka \\
obs18 & 2003.03 .03 & 52702.3 & Kolka \\
obs19 & 2003.03 .04 & 52703.3 & Kolka \\
obs20 & 2003.03 .05 & 52704.3 & Kolka \\
obs21 & 2003.04 .18 & 52748.5 & Kolka \\
obs22 & 2003.07 .31 & 52852.5 & Kolka \\
obs23 & 2004.02 .11 & 53047.3 & Kolka \\
obs24 & 2004.10 .14 & 53293.5 & Kolka \\
obs25 & 2005.07 .22 & 53574 & Lobel \\
obs26 & 2005.10 .11 & 53655.5 & Kolka \\
\hline & & & \\
& & &
\end{tabular}

and the turbulent velocity $v_{\mathrm{t}}$, we analysed the data of the different observations in an identical manner, to ensure the resulting values would be consistent and accurate, though in a relative, not absolute sense.

The analysis of the spectra was performed on an ensemble of measured lines and their equivalent widths. The observational data were compared to model equivalent widths obtained by interpolating equivalent widths in a grid of model spectra. The model spectra results were based on three premises: (1) the use of Kurucz LTE models; (2) the use of published atomic data, notably $\log (g f)$-values, preferably from NIST data (cf. references, Sect. 2.2), and in some cases from Kurucz line atomic data (cf. references, Sect. 2.2); and (3) the use of the model calculation program SCAN, developed in SRON (SRON Laboratory for Space Research) in Utrecht by L. Achmad, based on an earlier program Scan from Kiel, Germany, and modified by M. Burger at SRON (Nieuwenhuijzen \& De Jager 2000). Results derived by these three inputs LTE, NIST, and SCAN, have not been calibrated as a total package, so that the results can only be taken as relative in terms of accuracy.

In this paper, we use the value of the metallicity parameter derived in earlier analyses (cf. Luck 1975; Fry \& Aller 1975; Nieuwenhuijzen \& De Jager 2000), where it was found that abundances were close to the solar value. We use Kurucz stellar LTE models with solar abundances, thus limiting the research to three unknowns, viz. $T_{\text {eff }}, \log g$, and $v_{\mathrm{t}}$.

\subsection{Observations}

Obs5 and obs 25 were obtained with the SOFIN echelle spectrograph on the NOT, and obs 12 with the UES on the WHT, both at sites in La Palma. Obs6-obs11, obs13-24, and obs26 were obtained at the Tartu Observatory. Four earlier published spectra (Nieuwenhuijzen \& De Jager 2000) are given as obs1-obs4 (cf. Table 1). 
Table 2. Estimated errors in the $E W \mathrm{~s}$, with the exception of obs4 and obs 25 .

\begin{tabular}{lc}
\hline \hline Range & $E W$ Error \\
\hline $10 . . .30 \mathrm{~m} \AA$ & $20 \%$ \\
$30 . .70 \mathrm{~m} \AA$ & $10 \%$ \\
$70 . .200 \mathrm{~m} \AA$ & $5 \%$ \\
$>200 \mathrm{~m} \AA$ & $2 \% \ldots . .5 \%$ \\
\hline
\end{tabular}

Table 3. Grid of model photospheres used in the spectral line library.

\begin{tabular}{lccccc}
\hline \hline$T_{\text {eff }}(\mathrm{K})$ & $\log g$ & $v_{t} \mathrm{~km} \mathrm{~s}^{-1}$ & $\ldots$ & $\ldots$ & $\ldots$ \\
\hline $4250-6000$ & $0.0-2.0$ & 4 & 6 & 13 & 19 \\
$6250-7500$ & $0.5-2.5$ & 4 & 6 & 13 & 19 \\
$7750-8250$ & $1.0-3.0$ & 4 & 6 & 13 & 19 \\
$8500-9000$ & $1.5-3.5$ & 4 & 6 & 13 & 19 \\
$9250-9250$ & $2.0-4.0$ & 4 & 6 & 13 & 19 \\
\hline
\end{tabular}

The full set of observed equivalent widths (EWs) in units of $\mathrm{m} \AA$ are available in electronic Tables A.1.1 with columns for observations obs1-obs26, and rows for spectral lines with wavelength $(\AA)$ and element number.

\subsection{Spectral lines}

The spectral lines used in this research are given in Table A.2 in the form of wavelength, ele, ion (atomic number combined with ionization stage), elo(cm-1), $10 \log (g f)$, and an accuracy value based on NIST $^{1}$ data and Martin et al. (1988) publications in the form of a letter designation A-E, corresponding to accuracies of $10-50 \%$. Lines that were unavailable from NIST were taken from Kurucz ${ }^{2}$. These data were used with an accuracy factor of $50 \%$, as we did not have a critical compendium of the accuracy of these $g f$-values. We translated these figures into logarithmic values and added them as "accuracy" to the list in Table A.2.

\subsection{Errors in observations}

Errors in the observed EWs were estimated following Table 2, except for obs4 and obs25. For these, a different approach was taken, using one measurement to help determine the optimal continuum and two measurements with a $1 \%$ difference of continuum level. Multiplying this difference value by an estimate of the continuum accuracy provides a measure of the observational accuracy in EW. The errors are given in Table A.1.2, which has the same structure as that for the observed EWs.

\subsection{EW data from models}

A range of temperatures and $\log g$ 's was encountered in the analysis of the observations. We decided to use a grid of LTE models and for each model created a list of lines and modelled EWs. Table 3 defines the model grid. The data of the models is represented by a three-dimensional chebychev polynomial with a limited number of terms $N$. In our case, $N=45$. A library was

\footnotetext{
1 http://physics.nist.gov/PhysRefData/ASD/index.html http://physics.nist.gov/PhysRefData/datarefscover.html http://physics.nist.gov/cgi-bin/ASBib1/TransProbBib. cgi

2 http://cfaku5.cfa.harvard.edu/temp

http://kurucz.harvard.edu/grids.html
}

created to contain the polynomial terms for each observed spectral line. The three-dimensional interpolation procedure is based on our earlier two-dimensional chebychev codes (De Jager et al. 1988; cf. also Appendix B.1).

Thus, the parameters in the library contain information about EW-values over the complete grid for each spectral line.

The modelled EW-values for the optimal set of parameters in the fitting procedure are given in Table A.1.3, which has the same structure as that for the observed EWs.

\subsection{Accuracy of EW data from models}

The estimated errors in the modelled lines take into account the inaccuracy of the $g f$-values underlying the model EWs. If the accuracy value $\Delta g f$ is known, the influence of this value on the modelled EW is found by doing calculations for $g f$, $g f \pm \Delta g f$ and taking the mean difference of the EWs as an accuracy estimate $\Delta \mathrm{EW}$.

The accuracies of the modelled equivalent widths are determined for all the spectral lines and all models in the model grid (cf. Table 3). These data are then reduced to $N$ parameters for a chebychev polynomial evaluation of $\triangle \mathrm{EW}$ and added to the spectral line library. There are thus two separate entries in the library for each modelled spectral line.

The errors in the modelled EW-data for the optimal set of parameters in the fitting procedure are given in Table A.1.4, which has the same structure as that for the observed EWs.

\subsection{Comparing observational and model EW data}

For each observation, the analysis program compares observed and modelled EWs for all relevant spectral lines, together with a combined $\sigma_{\text {total }}$ derived from

$\left(\sigma_{\text {total }}\right)^{2}=\left(\sigma_{\text {obs }}\right)^{2}+A \cdot\left(\sigma_{\text {model }}\right)^{2}$.

We always took $A=1.0$. The total $\sigma_{\text {total }}$ is given in Table A.1.4.

Defining the "significance" as the difference EW-value divided by $\sigma_{\text {total }}$, we give the "significance" for each spectral line and the optimal set of parameters in the fitting procedure in Table A.1.5, which has the same structure as that for the observed EWs.

When the significance is more than one standard deviation, this may be due to either an outlier or a line misidentification (e.g. caused by varying temperatures when other lines appear to be predominant at about the same wavelengths). We discuss deviations for some lines in Table A.3.

In one case, we used a correlation technique to find the mean wavelength shift between the observed wavelengths and modelled lines in Table A.4.

To achieve "stable" results, we used a method of "robust fitting" with a Lorentzian tail distribution to ensure that large deviations in $\chi^{2}$ only marginally influence the fitting process (cf. Press et al. 1992, chap. 15.7; Vetterling et al. 1992). In this paper, we use the merit function of the Lorentzian minimization as a " $\chi 2$ " value.

\subsection{Results of analysis}

The results of the best-fit analysis are given in Table 4 and in Fig. 2 for $\log T_{\text {eff }}$, in Fig. 3 for $\log g$, and in Fig. 4 for the turbulent velocity $v_{\mathrm{t}}\left(\mathrm{km} \mathrm{s}^{-1}\right)$.

We note that when $\log g$-values are extrapolated outside the grid of model values of $T_{\mathrm{eff}}, \log g$, and $v_{\mathrm{t}}$ an asterisk is given in Table 4 before the value of $\log g$ to indicate extrapolation. 
Table 4. Results of LTE effective temperatures $T_{\text {eff }}$, Kurucz-log $g$ values, and turbulent velocities $v_{\mathrm{t}}$.

\begin{tabular}{lcccc}
\hline \hline $\begin{array}{l}\text { Obs } \\
\text { nr }\end{array}$ & $\begin{array}{c}\text { JD } \\
-2400000\end{array}$ & $\begin{array}{c}T_{\text {eff }} \\
(\mathrm{K})\end{array}$ & $\begin{array}{c}\log g \\
(\mathrm{~cm} \mathrm{sec}-2)\end{array}$ & $\begin{array}{c}v_{t} \\
\left(\mathrm{~km} \mathrm{~s}^{-1}\right)\end{array}$ \\
\hline obs1 & 41911.3 & $4913.7 \pm 72.0$ & $*-0.88 \pm 0.07$ & $11.67 \pm 1.39$ \\
obs2 & 43737.3 & $5601.7 \pm 120.7$ & $*-0.62 \pm 0.08$ & $13.10 \pm 0.99$ \\
obs3 & 45898.3 & $5334.0 \pm 239.4$ & $*-0.76 \pm 0.12$ & $14.07 \pm 1.54$ \\
obs4 & 49824.3 & $7346.2 \pm 70.6$ & $* 0.40 \pm 0.19$ & $14.23 \pm 1.20$ \\
obs5 & 51702.5 & $7611.6 \pm 69.5$ & $* 0.55 \pm 0.21$ & $11.20 \pm 1.00$ \\
obs6 & 51824.4 & $7777.7 \pm 73.3$ & $* 0.63 \pm 0.25$ & $10.46 \pm 0.98$ \\
obs7 & 51876.4 & $7623.5 \pm 61.7$ & $* 0.56 \pm 0.21$ & $10.96 \pm 0.94$ \\
obs8 & 51922.3 & $7747.1 \pm 60.4$ & $* 0.66 \pm 0.21$ & $9.75 \pm 0.87$ \\
obs9 & 51957.3 & $7812.1 \pm 65.9$ & $* 0.75 \pm 0.20$ & $10.61 \pm 0.98$ \\
obs10 & 51999.4 & $8024.9 \pm 76.9$ & $1.09 \pm 0.27$ & $11.19 \pm 1.16$ \\
obs11 & 52178 & $7619.0 \pm 59.9$ & $* 0.51 \pm 0.17$ & $9.99 \pm 0.80$ \\
obs12 & 52184 & $7534.4 \pm 67.7$ & $0.51 \pm 0.19$ & $11.14 \pm 0.97$ \\
obs13 & 52230.4 & $7894.6 \pm 80.9$ & $1.02 \pm 0.28$ & $9.91 \pm 0.98$ \\
obs14 & 52359.6 & $7893.3 \pm 83.8$ & $1.20 \pm 0.36$ & $10.30 \pm 1.09$ \\
obs15 & 52508.5 & $7868.2 \pm 80.4$ & $* 0.88 \pm 0.24$ & $11.90 \pm 1.09$ \\
obs16 & 52623.4 & $8019.2 \pm 82.6$ & $1.30 \pm 0.37$ & $11.84 \pm 1.22$ \\
obs17 & 52690.3 & $7639.2 \pm 85.3$ & $* 0.77 \pm 0.33$ & $9.88 \pm 1.14$ \\
obs18 & 52702.3 & $7576.0 \pm 70.9$ & $0.60 \pm 0.22$ & $9.57 \pm 0.89$ \\
obs19 & 52703.3 & $7525.1 \pm 66.4$ & $* 0.48 \pm 0.21$ & $9.49 \pm 0.96$ \\
obs20 & 52704.3 & $7550.3 \pm 69.0$ & $0.53 \pm 0.22$ & $9.43 \pm 0.85$ \\
obs21 & 52748.5 & $7894.7 \pm 84.1$ & $0.99 \pm 0.26$ & $11.95 \pm 1.27$ \\
obs22 & 52852.5 & $7909.8 \pm 82.6$ & $1.05 \pm 0.25$ & $12.46 \pm 2.06$ \\
obs23 & 53047.3 & $7942.2 \pm 82.7$ & $1.11 \pm 0.31$ & $11.15 \pm 1.21$ \\
obs24 & 53293.5 & $7998.5 \pm 70.8$ & $1.03 \pm 0.23$ & $11.22 \pm 1.39$ \\
obs25 & 53574 & $7639.0 \pm 77.2$ & $* 0.50 \pm 0.24$ & $11.19 \pm 0.96$ \\
obs26 & 53655.5 & $7907.2 \pm 80.0$ & $1.13 \pm 0.26$ & $10.00 \pm 0.88$ \\
\hline & & & &
\end{tabular}

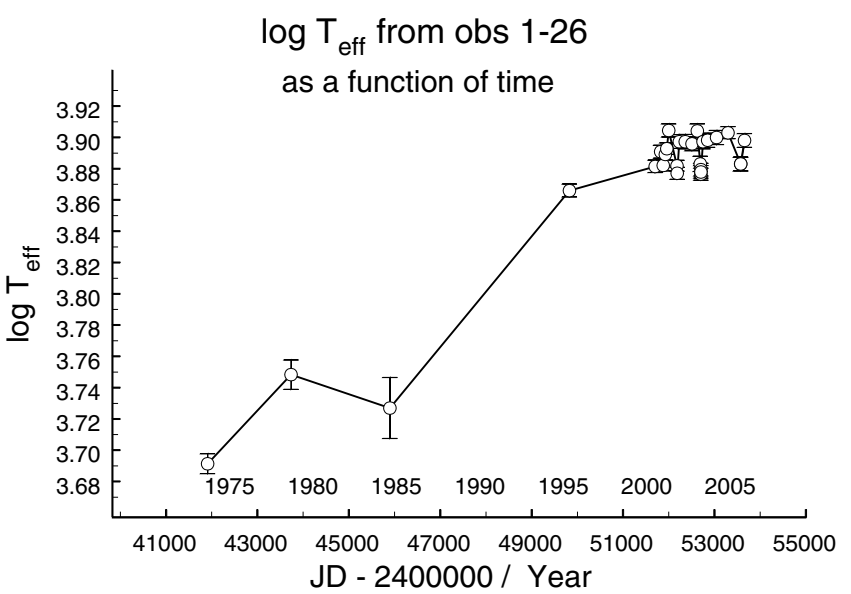

Fig. 2. Results of the fitting of $\log T_{\text {eff }}$ with Kurucz's LTE models for the observations obs1-obs26 as described in this paper.

\section{The analysis of colour observations}

We now analyse colour data. These are transformed with standard transformation tables and graphs into effective temperatures. A substitute for colour observations is the data on spectral type (MK classification). Not all data, especially the earlier data sets, are in the same photometric system, which is a problem that has to be corrected. In addition, the high interstellar reddening of HR 8752 demands information on the interstellar absorption. At the same time, the star itself is changing continuously in its various spectral parameters such as the spectral type, $B-V$ as well as in its $V$-magnitude. We have to determine the "intrinsic" or "unreddened" $B-V$ values that can be compared with MK spectral types, using the $T_{\text {eff }}$ data. To that end, information on the interstellar extinction parameter $E(B-V)$ is imperative.
Log g from obs 1-26

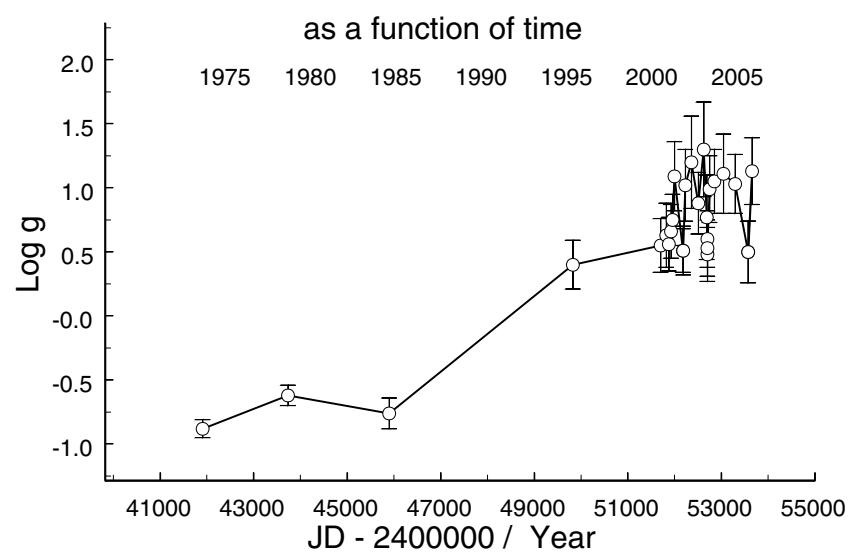

Fig. 3. Results of the fitting of $\log g$ with Kurucz's LTE models for the observations obs1-obs26 as described in this paper.

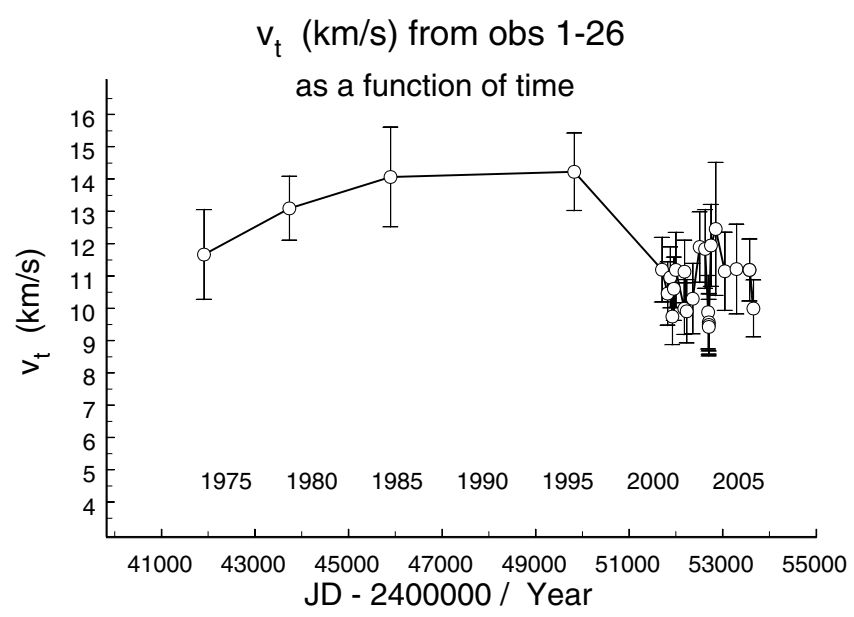

Fig. 4. Results of the fitting of $v_{\mathrm{t}}$ with Kurucz's LTE models for the observations obs1-obs 26 as described in this paper.

This section consists of three parts: 1) We determine the value of $E(B-V)$ from the overview of the colour- and MK-information below, and can then create a $T_{\text {eff }}$ time history. 2) An earlier set of multi-colour observations, together with the synchronization of the MK-type determination (by interpolation) leads to a separate (re)determination of $E(B-V)$ and allows us to determine $R$, the ratio of absorption in $V$-colour to $E(B-V)$. 3) When the distance is known, we can then estimate absolute magnitudes.

\subsection{B - V colour, MK-, and $T_{\text {eff-observations }}$}

The available $B-V$ information has been collected in a critical compendium (Table A.7) and in a combined, ordered database of all observations after about 1973 (Table A.8) with author reference included for each observation. (Note: our list of references also contains the data sources without any direct link in the main text.)

New observations are published in Table 5. This data is also included in Table A.8 under letter "n". Data on some early visual measurements is included as "early-colour", Table A.6.

In one graph, we use Tycho photometer data (cf. Nieuwenhuijzen \& De Jager 2000, with data on HR $8752=$ HIP 115 361, ESA 1997) from the HIPPARCos satellite. 
Table 5. $B-V$ Observations, period JD=2 448 813-2 449 299, Konkoly Observatory, Budapest.

\begin{tabular}{lcc}
\hline \hline Julian Day & $V$ & $B-V$ \\
\hline 2448813.532 & 5.088 & 0.872 \\
2448853.475 & 5.098 & 0.964 \\
2448859.374 & 5.113 & 0.973 \\
2448897.348 & 5.215 & 1.011 \\
2448904.309 & 5.202 & 1.001 \\
2448936.271 & 5.166 & 1.060 \\
2449005.252 & 5.136 & 0.973 \\
2449006.229 & 5.128 & 0.985 \\
2449254.380 & 5.095 & 0.894 \\
2449266.368 & 5.089 & 0.888 \\
2449289.231 & 5.087 & 0.903 \\
2449290.222 & 5.085 & 0.894 \\
2449292.269 & 5.097 & 0.892 \\
2449299.293 & 5.107 & 0.880 \\
\hline
\end{tabular}

Table A.7 gives an overview of the combined sequence of $B-V$ measurements from 1942-1974, followed by the "intrinsic" or $(B-V) 0$ value of Gyldenkerne (1955) for $E(B-V)=0.70$.

Table A.8 entitled " $B-V$-1976-data" $(B-V)$ observations gives an overview of the $V,(B-V)$ measurements in the period 1976-1993 from various authors. A letter code gives the author reference, where the letter "n" refers to the data published here. The table gives the observation date, $V$-magnitude, $(B-V)$, followed by columns with transformed values for $(B-V) 0$, $m_{\text {bol }}=V+B C$, spectral parameter s, $\log T_{\text {eff }}$, bolometric correction BC and $m_{\text {bol }}$, using Table B.2 "calibrate-hyper", and finally the author reference.

Table A.5 gives an overview of MK data from 1919-1987 with annotations and estimates of observing periods. The MK-data is transformed to $T_{\text {eff }}, B-V$, and bolometric correction $\mathrm{BC}$ by transforms described in Table B.2. In this analysis, we use the continuous spectral $s$-parameter introduced by De Jager \& Nieuwenhuijzen (1987). Transforms of some older spectral class observations to MK are described in Table A.5. Except in those cases where this is indicated, the estimated accuracy in $s$ is about from 0.05 to 0.06 spectral steps in MK.

The $T_{\text {eff }}$ data is derived from spectral types or s-parameter values. For the calibrations between the various types of measurement, we refer to "calibrate-hyper" in Table B.2, which discusses the calibrations that are specific to hypergiants Ia+ or Ia0.

The temperature data in our paper also consists of the following four measurements, two measurements made in 1969 Sept. 7 of $T_{\text {eff }}=5250 \pm 250 \mathrm{~K}$, and $1998 \mathrm{Aug}$. $4 T_{\text {eff }}=7900 \pm 200 \mathrm{~K}$ from Israelian et al. (1999), one in $1970\left(T_{\mathrm{eff}}=5030 \pm 171 \mathrm{~K}\right.$, from Schmidt 1972), and lastly an estimate of the lowest temperature measurement ( $T_{\mathrm{eff}}=4000 \pm 300 \mathrm{~K}$ by Luck 1975$)$. These measurements are indicated by an " $X$ " symbol in Figs. 5, 6, and 10. The 1969 spectrum was obtained by Smolinski at the Dominion Astrophysical Observatory, Victoria, Canada.

"Early-colour" observations with estimates of $T_{\text {eff }}$ and $B-V$ are reproduced in Table A.6. In comparing $T_{\text {eff }}$, we may first use the estimate of temperatures given there. Later in the paper, we use the $B-V$ information. With HR 8752's large interstellar absorption, the temperatures may be higher than indicated when correcting for the shifting of colour towards the red.

The colour observations started around 1940 and are not all in the Johnson $B-V$ format that we use as reference in this paper. After 1940, the measurements were made primarily in $B-V$. The $T_{\text {eff }}$ data and the data in Tables A.5-A.8 form the basis of this study.
$T_{\text {eff }}$ from MK \& spectral observations 1896-2005

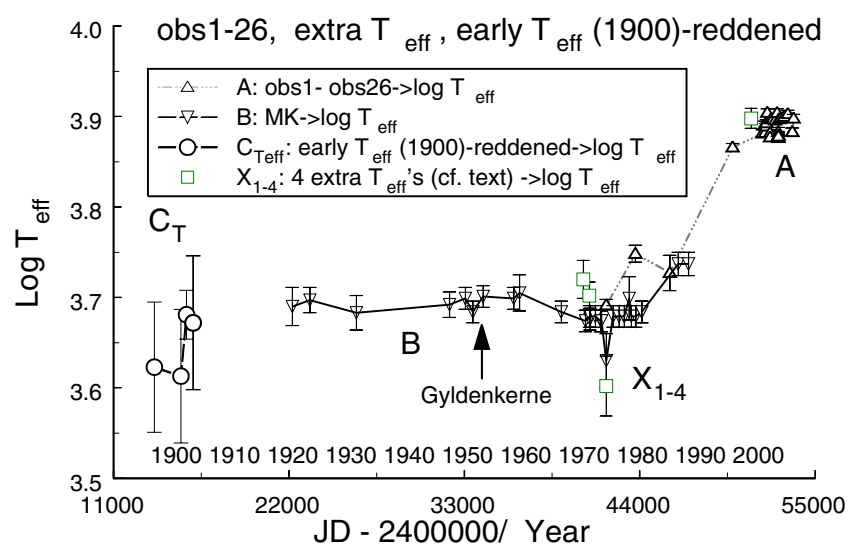

Fig. 5. Temperatures from $T_{\text {eff }}$ observations ("A"), from MK values transformed to $T_{\text {eff }}$ ("B"), from extra data ("X") and from temperature correlation data (" $\mathrm{C}_{T}$ )" form the base time-line of this study (step 1). For the meaning of the letters A-F, X we refer to the text.

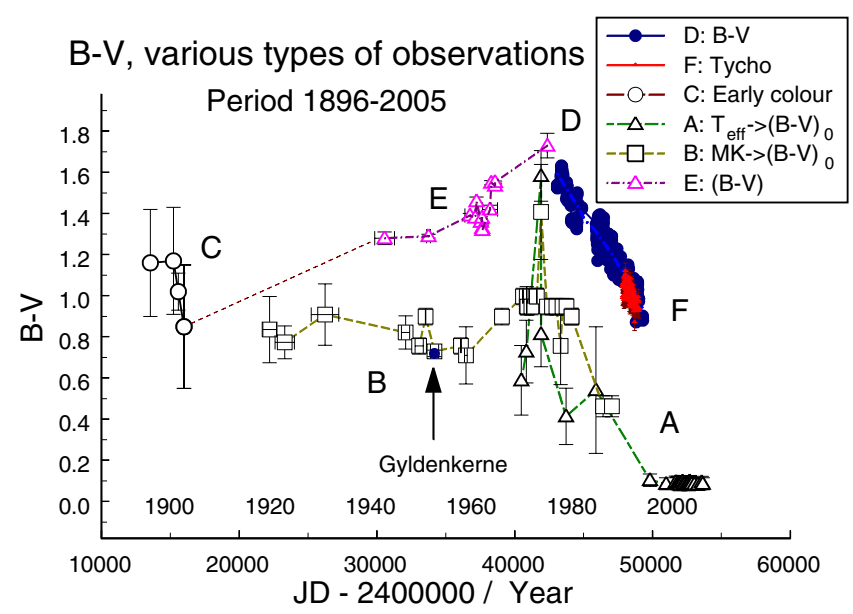

Fig. 6. The equivalent or "intrinsic" $B-V$ transformed from MK- ("A"), $T_{\text {eff }}$-observations ("B"), extra $T_{\text {eff- }}$-observations ("X") (cf. Fig. 5), and "early-colour" $B-V$ correlation data ("C") from step 1 are displayed together with (from step 2) "observed" $B-V$ 's for the period after 1973 ("D"). The 1942-1974 ("E") and Tycho data ("F") have been transformed to Johnson $B-V$, and have not been "unreddened". Note the influence of differential absorption caused by differences between Johnson and Tycho pass bands.

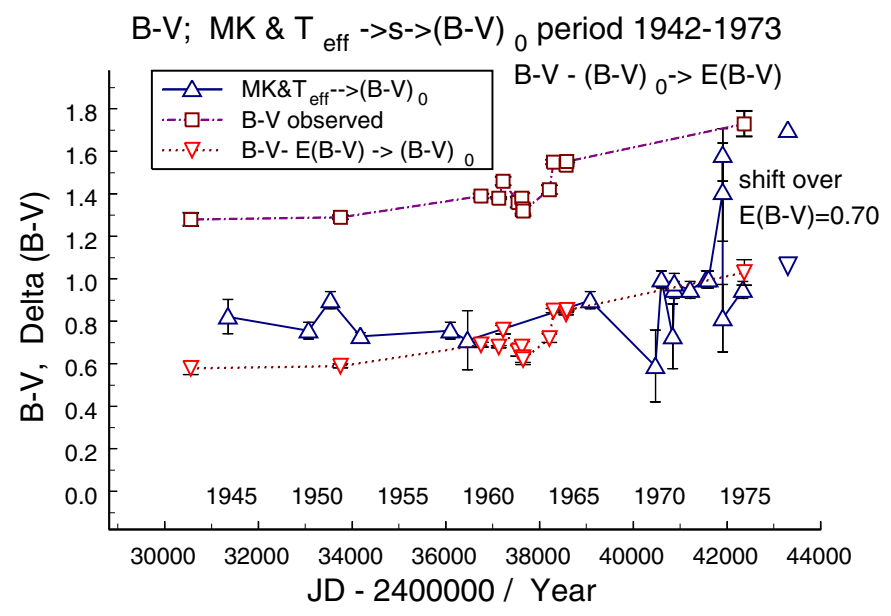

Fig. 7. Detail of left-hand part of Fig. 6, comparing reddened data directly with "intrinsic" $(B-V)$ 's from $\mathrm{MK}$ - and $T_{\mathrm{eff}}$-values. 
$B-V ; M K \& T_{\text {eff }}->S->(B-V)_{0}$ period 1973-2005

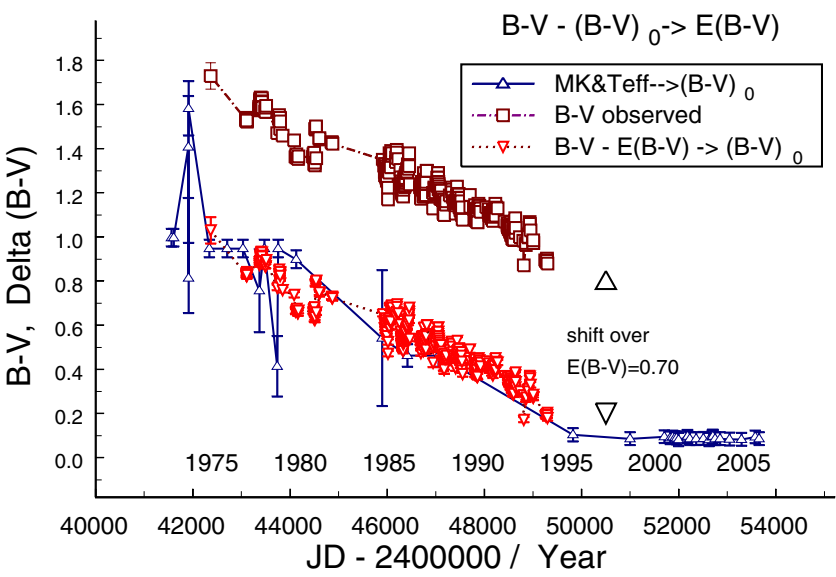

Fig. 8. As Fig. 7 for the right-hand part of Fig. 6. Taken together with Fig. 7, a downward shift of the reddened data over $\Delta(B-V)$ yields a "best" data fit with the "intrinsic" $(B-V)$ 's for $\Delta(B-V)=E(B-V)=$ $0.70 \pm 0.02$. This forms step 3 .

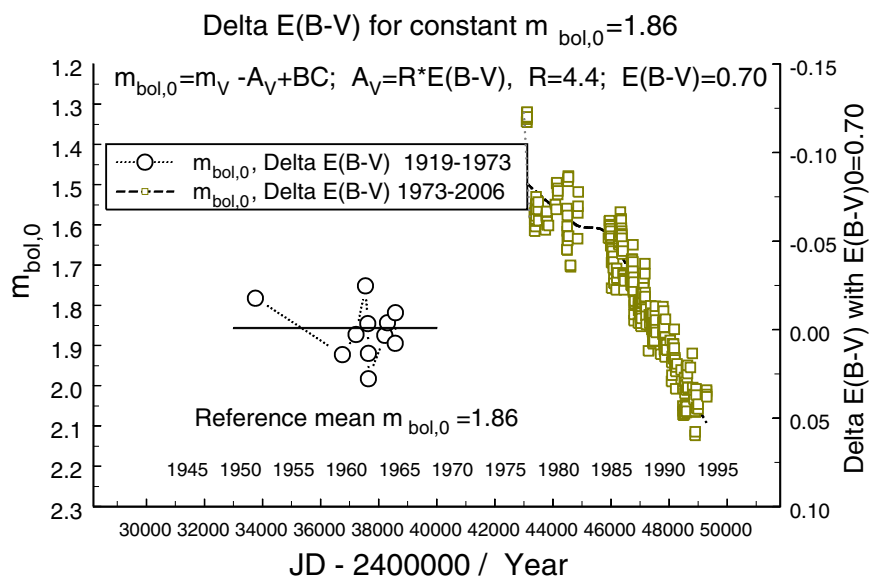

Fig. 9. The right-hand ordinate shows the $\Delta E(B-V)$-value needed to obtain a constant $m_{\text {bol }, 0}=1.86$. Relatively small differences in $E(B-V)$ appear to be needed. The left-hand ordinate displays the "unreddened" bolometric magnitude given as $m_{\text {bol }}=V-A_{\mathrm{V}}+B C$, with $A_{\mathrm{V}}=R \times$ $E(B-V)$, for the timeframe 1942-1996. The mean value of $m_{\mathrm{bol}, 0}$ for 1919-1973 seem to cluster around a value near to $m_{\mathrm{bol}, 0}=1.86$, while for 1973-1996 a larger range of values is involved. This forms step 4.

\subsection{Determining the interstellar extinction $E(B-V)$}

Values of $E(B-V)$ in the literature for HR 8752 vary from 0.27 to 0.82 . In this paper, we try to get a direct measure by comparing between the timelines of the observed $B-V$ values and the $T_{\text {eff }}$ and MK data transformed to $T_{\text {eff }}$ data, and then transform them to "intrinsic" $B-V$ values, i.e. without the effects of interstellar absorption.

This procedure is performed in the following five steps:

Step 1: transform MK data to $T_{\text {eff }}$; carry these over to the

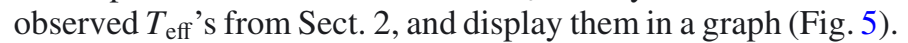
Transform the various data to $B-V$, and display this in step 2 (Fig. 6, lower line).

Step 2: add the observed $B-V$ data to the graph (Fig. 6, upper line).

Step 3: compare the two time segments of the graph (Fig. 7, Fig. 8) and find a value for the difference $\Delta(B-V)$ such that a reasonable overlay is obtained.

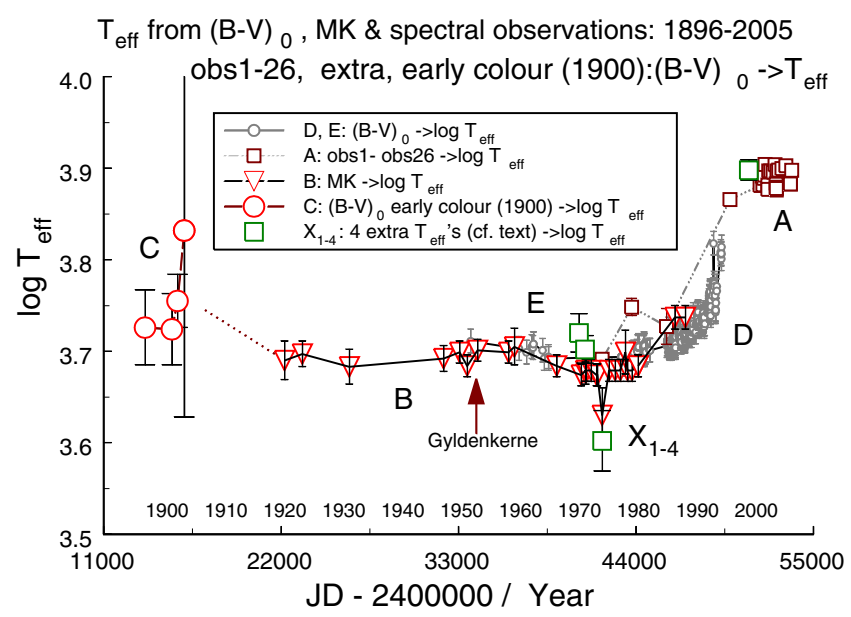

Fig. 10. Combination of the temperatures in one plot: from $B-V$ corrected for interstellar extinction, from $\mathrm{MK} \rightarrow T_{\text {eff }}$, from obs1-obs26 (Sect. 2), and from some extra data (indicated by " $X$ " $\left(X_{1-4}\right)$, cf. text). The temperatures derived from the $B-V$ and MK data combine reasonably well. The temperatures for obs1-obs2, obs4-obs6 also seem to follow the combined data, and the values for obs7-obs26 extend the data. The difference from obs 2 is relatively large. This forms step 5.

Step 4: check the measured value by verifying the value of $E(B-V)$ required at any time to obtain a constant "unreddened" light output $m_{\text {bol, } 0}$ in Fig. 9, (with known $R$ ). We compare the identified value to literature values.

Step 5: correct the $B-V$ observations for interstellar absorption, transform the now "intrinsic" values to $T_{\text {eff }}$, and display the $\mathrm{MK} \rightarrow T_{\mathrm{eff}}$, the $T_{\mathrm{eff}}$, and the $B-V->T_{\mathrm{eff}}$ values along one timeline (Fig. 10). Compare this with the "early-colour-data" $T_{\text {eff }}$ (cf. Table A.6).

The determination of $E(B-V)$ is done in the first three of the five steps mentioned at the beginning of Sect. 3. We also perform a direct determination from available colour data and an interpolation of MK-data for observing date and discuss the results in steps 4 and 5.

Step 1. The temperature data from 1896 to 2005 are presented in Fig. 5. The $T_{\text {eff }}$ values are given for MK-data ("B",

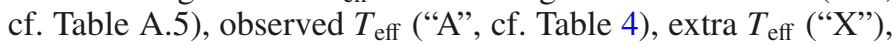
and the "early-colour" temperature correlation data (" $\mathrm{C}_{\mathrm{T}}$ ", $\mathrm{cf}$. Table A.6). The " $\mathrm{C}_{\mathrm{T}}$ " data have to be corrected for reddening and are expected to be somewhat higher than given. The " $C$ " data for "early-colour", experimentally corrected for interstellar reddening with $E(B-V)=0.70$ shows (cf. Fig. 10) a somewhat higher temperature. In Fig. 5 at "B" and "A", the different types of temperature measurements ( $\mathrm{MK}$ and spectral models) can be compared. The error bars of the two types seem to overlap at nearly coincident (synchronous) timeframes, and although ob-

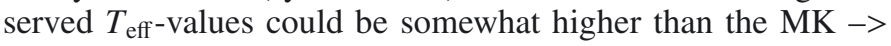
$\mathrm{s} \rightarrow>T_{\text {eff }}$ values (but for those at the time of the minimum around JD $24419000, \approx 1973$ ), we consider the agreement between the two types as reasonable. We suggest that the temperature is possibly $\log T_{\text {eff }}=3.65$ around 1900 , about $\log T_{\text {eff }}=3.68$ around 1925 , and $\log T_{\text {eff }}=3.7$ from 1940-1960 with dramatic changes between 1960-1980 and a sharp increase to $\log T_{\text {eff }}=3.9$ during 1990-2000. The measurement of Gyldenkerne (Gyldenkerne 1955, 1958; Strömgren \& Gyldenkerne 1955) is incorporated from $B-V \rightarrow T_{\text {eff }}$ using a $1 / 5$ sensitivity of influence of $E(B-V)$ with first approximations of $E(B-V)$, but is actually used in Fig. 6 as an intrinsic $(B-V)$ value. It is indicated by an arrow in the figure. 
A\&A 546, A105 (2012)

Table 6. Characteristics of four types of variations in the observations, cf. also Figs. 6, 11, and Sect. 5.6.

\begin{tabular}{lcccc}
\hline \hline Type & Days/years & Variation & Reference & Year \\
\hline 1 & $300-500 \mathrm{~d}$ & sawtooth & $1975-1995: \mathrm{D}, \mathrm{F}$ & $1940-1995$ \\
2 & $2-3 \mathrm{y}$ & smaller episodes & 1960: E, B, 1980: D, A & 1960,1980 \\
3 & $20 \mathrm{y}$ & special episode & 1973 minimum $T_{\text {eff }}$ & $1965-1980$ \\
4 & $100 \mathrm{y}$ & incomplete & Geyser-model? & before 1840-1973? \\
\hline
\end{tabular}

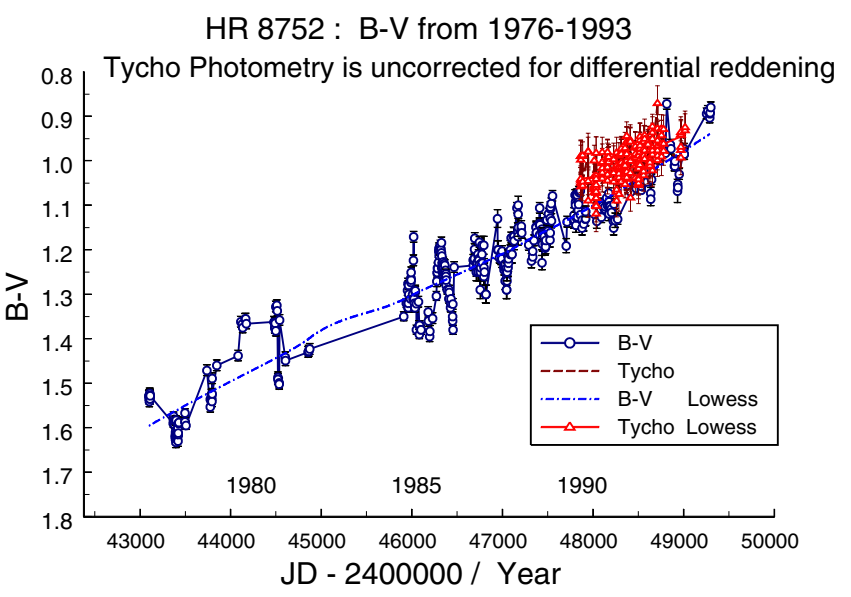

Fig. 11. Detail of $B-V$ and Tycho photometry observations showing "saw-tooth" variations with an amplitude of $\approx 0.2 \mathrm{~m}$, and a sequence time of a few hundred days. The difference between the Tycho and $B-V$ mean values is due to differential pass-band reddening; the Tycho data points have been obtained at small time differences and may indicate a field of shockwaves.

In Fig. 6, the $T_{\text {eff }}$ values from Fig. 5 have been transformed to $B-V$ (lower curves) for MK-data ("B") and observed $T_{\text {eff }}$, ("A"). From the "early-colour" data the $B-V$ correlation data are displayed ("C"). The "C" data have been corrected for reddening; in the first instance, one could expect an excess colour that is near to the value of $E(B-V)$ found later in this study, but this is uncertain. We note that the measurement of Gyldenkerne (1955, 1958) lies in a reasonable position of temperature and $B-V$ values, after having been corrected for an appropriate excess, cf. the discussion in Table A.7.

Step 2. In Fig. 6, we now add the observations from " $(B-V)$ 1973-data" ("D", cf. Table A.8), from " $(B-V)$-1950-data" ("E", cf. Table A.7) and finally the (transformed, still reddened) Tycho $B-V$ data ("F", cf. Nieuwenhuijzen \& De Jager 2000). These data lie in the upper part of Fig. 6. The Tycho data has been transformed to Johnson $B-V$ representation and has not been corrected for interstellar absorption. Some differential absorption is visible due to differences between Johnson- and Tycho passbands. The Tycho data set coincides closely in time with the " $(B-V)$-1973-data" ("D", cf. Table A.8). In this paper, we do not apply the somewhat complex dereddening procedures for the Tycho data.

Leaving aside the plot named " $C$ ", we characterize the " $\mathrm{B}$ "-, "A"-, and "X"-plots as indicating initially a medium temperature, after which the star becomes cooler with a minimum around 1973 followed by a growth toward higher temperature in " $D$ ", and continuing in nearly a straight line in section "F". The time between about 1965 and 1980 may indicate a special episode in the star's evolution (type 3, cf. Table 6 for an overview of four types of variations that we identify in Figs. 6 and 11).

There also seem to be shorter episodes around 1960 in "E" and "B" and around 1980 in "D" and "A" (which we indicate as type 2). These deviations are possibly not the semi-regular "sawtooth" variations seen in Sects. "D" and "F" (type 1), but they seem to be larger-scale, longer-time duration disturbances that influence the atmospheric parameters. Finally, there is an overall structure (of changes $\approx$ instability?) with a timescale of more than a century (100 years) (type 4$)$.

In the approach outlined below, we assume a constant bolometric magnitude to test and limit the choices of values of $E(B-V)$. For this reason we employ data for which simultaneous $B-V$ - and $V$-magnitudes are available. These data are effectively only available in the time range 1942-1996.

Step 3. In Fig. 6, the curves "E" and "D" are at some distance from the curves "B" and "A". For time-synchronous data, this distance defines the excess $E(B-V)$. To get a better view, we combine the data as two streams called the $B-V$ data (upper graphs) and "intrinsic" data (lower graphs), and split the timeline into two parts (Figs. 7, 8). A shift over $E(B-V)=$ $0.70 \pm 0.02$ brings a reasonable correlation with the observed $B-V$ values over the intrinsic $B-V$ values. We use this latter value in our paper, but first do a test to see where there are differences and if they depend on changes in bolometric magnitude over time. This value is also found when synchronization with interpolation is used to correct the $E(B-V)$ published by Johnson (mentioned below as a direct determination). However, the difference measurement given here is applicable to all available data. We from hereon use $E(B-V)=0.70 \pm 0.02$.

We also note the difference of about $0.15 \mathrm{~m}$ between the two data streams for the observations before 1960, the dips between 1960-1965, between 1970-1980, and the smaller bump (negative) difference between 1985-1995.

Step 4: Checking $E(B-V)$ in the case of a constant bolometric magnitude: Here we use the concepts and values of $R$ and $A_{\mathrm{V}}$ developed and determined in the next subsection. In Fig. 9, we show how much variation in $E(B-V)$ is needed to obtain a constant value of $m_{\mathrm{bol}, 0}=V+B C-A_{\mathrm{V}}$, i.e. assuming that the "unreddened" light output of the star stays constant. The energy output also consists of internal-, movement-, and perhaps other energies, and we wish to check the constancy of $E(B-V)$ and $A_{\mathrm{V}}$. It appears that only relatively small variations in $\Delta(B-V)$ around $E(B-V)=0.7$ are needed to obtain a constant value of $m_{\mathrm{bol}, 0}$ for the data clustering around the $E(B-V)=0.70$ line. After a number of trials, it appears that a constant value of $m_{\mathrm{bol}, 0}=1.86$ gives results that can be accurately compared with our observations of $\Delta(B-V)$ to find $E(B-V)$.

We define the "mean" of the "early observations" during 1950-1965 as a "mean" reference value with which other observations should be compared. We use this value in various places throughout this paper. Their averages can be given as $V=5.11$, $V_{0}=2.03, m_{\text {bol }}=4.94$, and $m_{\text {bol }, 0}=1.856$.

We note that the observations show a structure about the "mean" line: points below the line have less or negative absorption $\left(A_{\mathrm{V}}=R \times E(B-V)\right)$, while points above the line have more absorption than the mean value. These changes indicate that some form of energy interaction is taking place in the processes before, during and after the 1965-1980 episode. This exercise 
also shows that the derived value for (constant) $E(B-V)$ is a useful starting point.

We conclude that the apparent changes in bolometric magnitude are due to changes in the luminosity, and that $A_{\mathrm{V}}$ is to first approximation constant. We have indeed two possibilities:

1) Either we have the colour excess decreases by 0.05 between 1978 and 1995 with a constant luminosity or

2) We have a constant $E(B-V)$ and a variation in $L$.

It is unclear whether the grain distribution and the grain sizes close to the star could experience enough change over that period of time for possibility 1) to apply. It is similarly unclear whether the large increase in $T_{\text {eff }}$ as more UV radiation is emitted could affect the grain properties or whether any difference in absorption could be caused by matter ejection. For the time being, we decided to use option 2) above.

It also remains unclear whether modelling of the $R$-ratio for the extremely large changes in the atmosphere of HR 8752 might not lead to some change in $A v$. It seems that both later types and higher luminosities can correspond to higher $R$-ratios, as demonstrated by Fig. 8 of Crawford \& Mandwewala (1976), cf. also Table A.9.

Step 5, the last step in finding $T_{\mathrm{eff}}$ : determining the $\boldsymbol{T}_{\mathrm{eff}}$ variation over the twentieth century. Transformation of $B-V$ to temperature can now be done with the "unreddened" or "intrinsic" $(B-V)_{0}$ from the observed $B-V$ as $(B-V)_{0}=$ $B-V-E(B-V)$ using the transforms in Table B.2 to transform $(B-V)_{0} \rightarrow \mathrm{s}$, and $\mathrm{s} \rightarrow T_{\text {eff }}$ and finding the appropriate errors caused by measurement errors and transformation errors. Figure 10 shows the equivalent temperatures $T_{\text {eff }}$ transformed from $B-V$, together with $T_{\text {eff }}$ from obs1-obs26, the MK classifications, and the early-colour $B-V$. Both MK- and $B-V$ transformed temperatures give nearly identical temperature information, while the star is continuously changing. The data obs3-obs8 show how the temperature at the end of the $B-V$ observations rises extremely rapidly until the flattening out at the end of the observations at about $\log T_{\text {eff }}=3.9$. Obs 2 indicates a jump in temperature. In the plot, the early colour $B-V$ information is transformed to temperature using the same value of $E(B-V)$ as for the other data. The temperatures are higher than those mentioned in Sect. 3.1.2 and step 1 for the temperaturecorrelation data (cf. Fig. 5).

Here the position of the measurement by Gyldenkerne is indicated by an arrow. The four extra temperature measurements are indicated by four blocks (" $\mathrm{X}_{1-4}$ "). The first measurement is somewhat high (Israelian et al. 1999), the second (Schmidt 1972) and fourth (Israelian et al. 1999) are in the line of the other observations, and the third (Luck 1975) shows an estimate of the low temperature.

Figure 11 shows relatively large saw-tooth variations in $B-V$ observations with a magnitude of $\approx 0.2 \mathrm{~m}$, with various frequency components and time-spans. The Tycho observations show large variations over all measurements, probably indicating shockwaves going through the stellar atmosphere. The small offset visible in the Tycho data is due to differential reddening of the differing pass bands of the Tycho photometer. The ordinate is inverted with respect to Figs. 6-9 to comply with the usual plots. The two Lowess curves in Fig. 11 characterize the running means for the $B-V$ and the Tycho data. The term Lowess refers to a method for smoothing scatter plots with the help of robust locally weighted regression, (cf. Cleveland 1979, 1985; Cleveland \& Devlin 1988).

\subsection{Direct determination of $E(B-V)$ and $R$-ratio}

A direct measurement is also available from colour observations of HR 8752 (Johnson 1968) by comparing with an (interpolated) MK-spectral type, instead of using the (classic value) MK type G0 as a reference. To synchronize the observing dates for the colour and the MK-observations, we interpolate for the date JD 2438300 (27 Sept. 1963) in the sequence of MK measurements in our Table A.5 (codes 3:-6a:) between JD 2 436 462-2 439080 for MK G0Ia and G3 respectively, to get spectral type G2. When combined with the calibration data from Schmidt-Kaler (1982, Sect. 4.1.2.1), and the observations of Johnson (1966, Table 4), this leads to $E(B-V)=0.70 \pm \approx 0.02$.

The two techniques to time-synchronize the different types of observations result in the same number for $E(B-V)$ and enhance our confidence in the measured value.

Literature values: apart from Johnson (1968), there are two direct determinations of $E(B-V)$, by Kron (1958) with $E(B-$ $V) \approx E(P-V)=0.53$, transformed to $E(B-V)=0.58$ (Fernie 1972) and by Schmidt (1972) with $E(B-V)=0.66$. This last value is practically identical to the value we find here.

Another result is that with the given time-synchronization the other observed colour excesses can be redetermined, leading to a direct determination of the $R$-ratio, thus with known $E(B-V)$ the value of $A_{\mathrm{V}}$. The corrected $R$-ratio is $R=4.4 \pm \approx 0.2$ (cf. Table A.9 for details of the correction of the various colour excesses and the graphical determination of the $R$-ratio $R$ ).

Hence, $A_{\mathrm{V}}=R \times E(B-V)=3.08 \pm 0.16$. The "unreddened" visual and bolometric magnitudes are $V_{0}=V-3.08$ and $m_{\mathrm{bol}, 0}=$ $V+B C-3.08$, respectively.

We note that the high value for the $R$-ratio contrasts with the values of $R=3.0$ to 3.1 that are usually attributed to O, B stars (cf. e.g. Fitzpatrick \& Massa 2007). There are two possible reasons for this:

1) This high value (if it is true) points unambiguously to a non-canonical IS reddening curve along the line-of-sight towards HR 8752 whatever the reason for that (it may well be the parameters of dust close to HR 8752). In addition, we note the result of Johnson (1968) who found strong variations in the line-of-sight reddening for different regions in Cepheus, a result that should indeed be the topic of a separate investigation.

2) The variation in the $R$-ratio with spectral class/ type found by some studies, from combining stellar energy fluxes for various spectral types with filter transmission curves, as presented in Table A.9.

We note that the above values for $R$ and $A_{\mathrm{V}}$ are used in the previous subsection at the start of step 4 .

\subsection{Absolute magnitude}

Following a redetermination of the distance of HR 8752 (Van Leeuwen 2007) giving a parallax $\pi=0.73 \pm 0.25$ (milliarcsec), distance $\log d=3.137 \pm 0.149$ (parsec), and a distance modulus $m-M=10.685 \pm 0.746$, the absolute values for the "mean" reference is $M_{\mathrm{V}, 0}=-8.65 \pm 0.75$ and $M_{\text {bol, }, 0}=-8.82 \pm 0.75$.

The spectral parameter $s$ for the "mean" reference is $s=$ $4.976 \pm .068$ corresponding to a MK classification of F9-G0.5 with $M_{\mathrm{V}}=-8.9 \pm \approx 0.03$. Schmidt-Kaler (1982, section 4.1.2, Table 13) found for the absolute magnitudes of the MK system of class $\mathrm{Ia} 0$ that $\mathrm{F} 0-\mathrm{F} 8 \rightarrow M_{\mathrm{V}}=-9.0, \mathrm{G} 0 \rightarrow M_{\mathrm{V}}=-8.9$, and $\mathrm{G} 2 \rightarrow M_{\mathrm{V}}=-8.8$. We note that the "mean" reference 


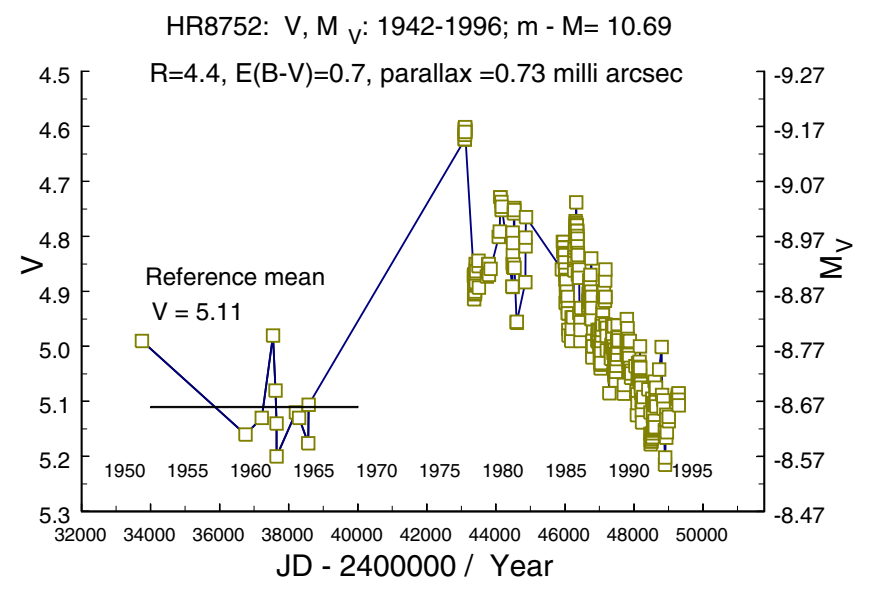

Fig. 12. The visual observed resp. absolute magnitudes $m_{\mathrm{V}}$ and $M_{\mathrm{V}}$ are given for the timeframe 1942-1996 as one plot with two ordinate values. The absolute visual magnitude is given as "unreddened" $M_{\mathrm{V}}=V-A_{\mathrm{V}}-(m-M)$, for $A_{\mathrm{V}}=R \times E(B-V)$ with the observed values of $R$ and $E(B-V)$. The mean value of $V$ for 1919-1973 appears to cluster around a value near $V=5.1$ and is indicated by a line at the left lower side of the figure.

we indicate here is thus compatible with a MK value of $\mathrm{MK} \approx$ $\mathrm{G} 0-\mathrm{Ia} 0 \pm \approx 0.7$ spectral step.

For the Galactic supergiant HR 8752, Mantegazza (1988, 1991, 1992, Eq. (4) resp. Eq. (5)) gives absolute visual magnitudes of $M_{\mathrm{V}}=-8.87 \pm 0.53$ and $M_{\mathrm{V}}=-9.04 \pm 0.34$. The MK spectral class of F7-Ia0 was measured on September 22, 1987, while the CP-indices were measured over December 15-18, 1987, to ensure that time synchronization between the spectral type and CP-index has been achieved. This spectral class is also near to that of the "mean" reference given above, so that a near comparison can be made.

Within the measurement errors, the different observations are both consistent with each other and with the data of the MK reference system for the luminosity class Ia0. This enhances the confidence in the combination of values found for $E(B-V), R$, $A_{\mathrm{V}}$, and distance modulus $m-M$.

We note that it is possible the luminosity given by our value for $M_{\mathrm{V}}$ is somewhat low. The $M_{\mathrm{V}}$-value depends directly on the value of the the "mean" reference of the "early observations" from 1950-1965 as defined earlier and may depend on how that was derived. We tried to find a meaningful value for the absolute magnitudes when the star varies strongly in time, and other processes exist that use or give extra energy in the time line of the star.

\subsection{Extending the $\mathrm{V}$-coverage back to 1840}

In Fig. 12, we give the values of the visual- and visual absolute magnitude as a function of time, during the timeframe 1942-1996.

Extending the 1942-1996 $V$-data backwards in time with data from Zsoldos (1986a, Table I) and adding three data points from the literature (cf. end of Table A.5) leads to Fig. 13. The "mean" reference value (Sect. 3, step 4) for $V$ is indicated by short horizontal lines within the period 1942-1996 in both Figs. 12 and 13. It seems that the process time for the indicated changing situation is longer than the time of the record, more than 150 years.

The $V$-observations indicate a visual brightness growing from below $V \approx 6$ to around $V \approx 5.1$ in some 60 years. It seems

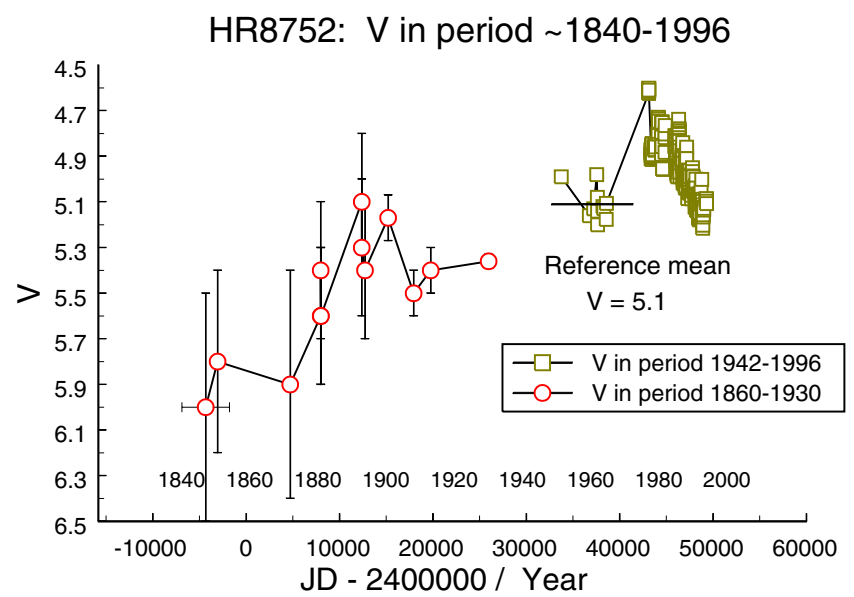

Fig. 13. Long time visual magnitude as a function of time, in the timeframe 1840-1996. The "mean" reference value (Sect. 3, step 4) for $V$ is indicated by a short horizontal line within the period 1942-1996.

that before 1840 the star was invisible to the naked eye. We quote from Zsoldos (1986a): "It is interesting that HR 8752 is not included in several catalogues compiled mainly before 1850 . The star has neither a Flamsteed number, nor a Bayer letter. It is not in the catalogues of Piazzi or Baily. Several reasons can be mentioned to explain why the star is not in these catalogues: (i) the star was fainter than $6 \mathrm{~m}$....... Bearing in mind Fig. 1, I suggest that the most likely explanation is the first possibility". This implies that the star was fainter than the sixth magnitude.

Are these variations due to a form of $\Gamma_{1}$-instability? We carry this question forward to the next section.

The main conclusions of this section can be summarized as:

1) We have derived a time-history of $T_{\text {eff }}$ (cf. Fig. 10) that allows us to determine:

2) Four different timescales (cf. Table 6) identified and discussed in Sect. 5.6.

3) That measurements $E(B-V), R$, and $A_{\mathrm{V}}$ can help us to measure absolute magnitudes for a subset of the data (cf. Sects. 5.2 and 6).

\section{Atmospheric (in)stability and the yellow evolutionary void}

To understand the evolutionary scenario of HR 8752 and other hypergiants, we start from models of Meynet et al. (1994), which form part of a series of evolutionary models with different authors. In the following, we use unpublished evolutionary data from one of us (GM) and models from Meynet et al. (1994) with rates of mass-loss twice as high as the mass-loss given in De Jager et al. (1988). The factor of two may seem arbitrary but was taken to achieve the best mean time fit of models compared to observations. The mean time fit is the average of the total mass-loss, hence includes "regular" mass-loss during "stable" periods of mass-loss and periods of massive loss of mass during a time of "instability".

We note that this "instability" mass-loss occurs during the evolution of the models along track 4 , and not along track 3 . The text below refers to track 4 unless otherwise indicated.

We now empirically check the condition $\left(3 \Gamma_{1}-4\right)<0$ for a stellar model traversing the region of the yellow evolutionary void. As an example for this study, we take the Meynet et al. (1994) model m040e020 that gives $L, T_{\mathrm{eff}}$, mass, and mass-loss as a function of age to find the physical parameters including $\Gamma_{1}$ 
Meynet model m040e020, Track 4

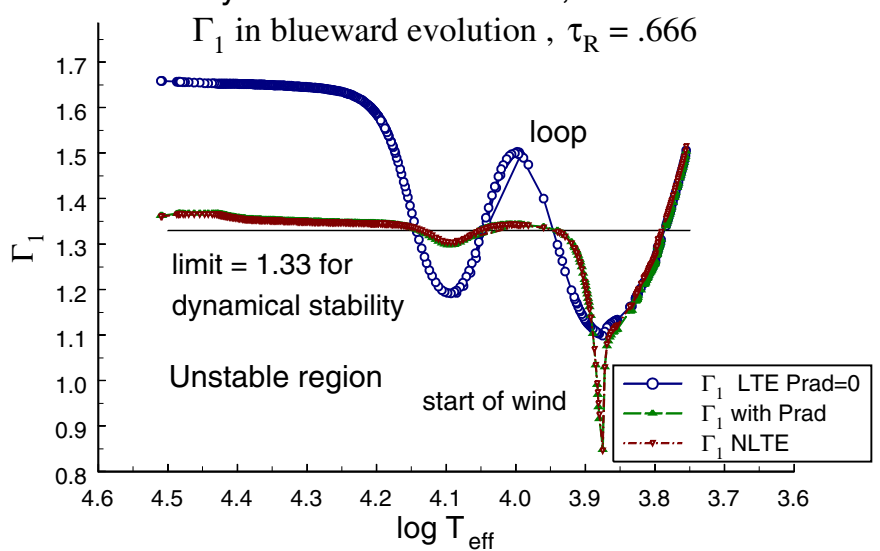

Fig. 14. In the atmosphere, the factor $\left(3 \Gamma_{1}-4\right)<0$ can give rise to dynamical instability (cf. Ledoux 1958, 1965) when this factor is negative. Using data from Meynet models (Meynet et al. 1994, and priv. comm. 1997) and code from Nieuwenhuijzen \& De Jager (1995), the value of $\Gamma_{1}$ during the evolution of m040e 020 is calculated for $\tau_{\text {Ross }}=2 / 3$ (at the "radius" of the star) and plotted against effective temperature, using the same direction as shown in the HR-diagram. The dynamical instability limit is indicated: $\Gamma_{1}$ values below this limit can give rise to dynamical instability if the layer where this value is negative is "large" enough (Ledoux 1958, 1965).

\section{Meynet model m040e020, detail of Track 4}

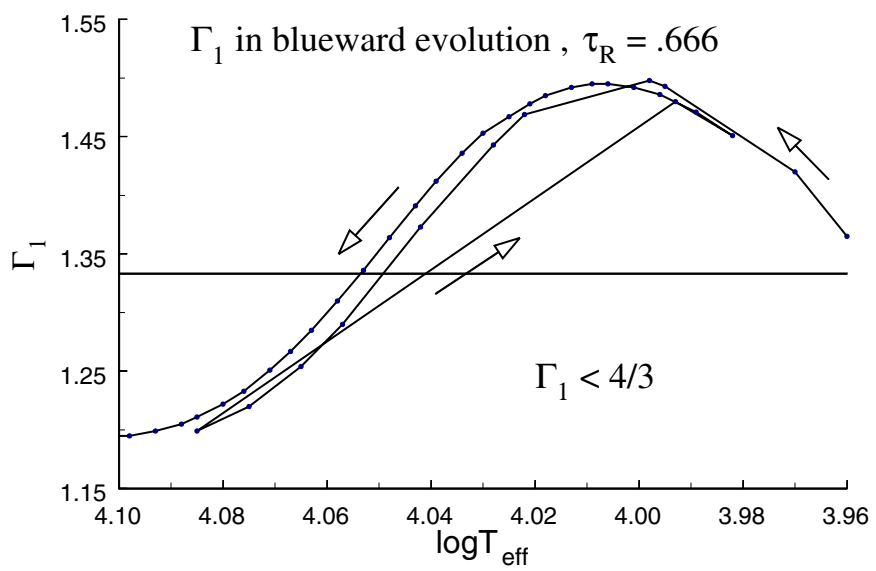

Fig. 15. Detail of Fig. 14 with a loop in the evolutionary computations. These diagrams show $\Gamma_{1}$ as a function of increasing temperature (evolution goes here from right to left. Figure 14 can be compared with the area of the yellow evolutionary void in Fig. 1.

at an optical depth of $\tau_{\text {Ross }}=2 / 3$ (the stellar radius) and lower values down to $\tau_{\text {Ross }}=0.03$. We use a code by Nieuwenhuijzen \& De Jager (1995) based on statistical data of the shock strength given in that paper and Nieuwenhuijzen et al. (1994). The code for the different forms of $\Gamma_{1}$ is taken from Lobel et al. (1992) and Lobel (2001). The influence of non-LTE effects on $\left\langle\Gamma_{1}\right\rangle$ are discussed in Lobel (2001, where $\left\langle\Gamma_{1}\right\rangle$ is defined in Eq. (68)). We use Lobel's definition in our Sect. 4.1, Eq. (1). Atmosphere models of yellow hypergiants with $T_{\text {eff }}$ between $6500 \mathrm{~K}$ and $7500 \mathrm{~K}$ have deep $\left\langle\Gamma_{1}\right\rangle$ minima below $4 / 3$ over a large fraction of the atmosphere, which strongly diminish their overall dynamic stability.

For the model m040e020, we show in Fig. 14 the variation in $\Gamma_{1}$ as a function of temperature, and in Fig. 15 a detail of a loop returning from $\log T_{\text {eff }}=4.08$ to 3.98 , and then returning unimpeded to higher temperatures, and in Fig. 16, from the

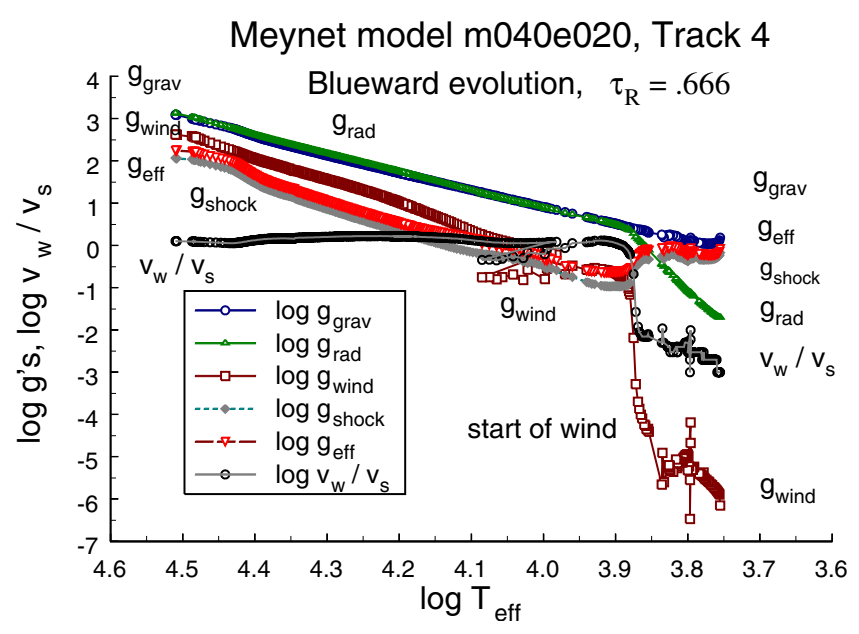

Fig. 16. Various accelerations in the atmosphere for the same model and their resulting $g_{\text {eff }}$. In general, $g_{\text {rad }}$ and $g_{\text {grav }}$ are very close to each other, and around $\log T_{\text {eff }} \approx 4.3$ the value of $g_{\text {rad }}$ is even somewhat larger than $g_{\text {grav }}$, the wind growing to compensate. We note the onset of the wind at low temperatures.

(above) acceleration model calculations, the acceleration parameters $g_{\text {grav }}, g_{\text {rad }}, g_{\text {shock }}, g_{\text {wind }}$, and $g_{\text {eff }}$, for newton-, radiative-, shock-, wind-, and effective-acceleration, respectively, together with the ratio of wind velocity $v_{\text {wind }}$ to sound velocity $v_{\mathrm{s}}$. In these three graphs, the temperature is not an unambiguous function of time, as can be seen for the "loop" in Figs. 14 and 15 and the "loop" for $g_{\text {wind }}$ in Fig. 16.

The parameter $\Gamma_{1}$ is presented in three forms: LTE with $P_{\text {rad }}=0$, LTE with $P_{\text {rad }}$, and NLTE. There is no difference between LTE with $P_{\text {rad }}$ and NLTE for $\tau_{\text {Ross }}=2 / 3$. The curve for $P_{\text {rad }}=0$ is visually more pronounced and gives a good insight into the possible area of instability where $\Gamma_{1}$ may be less than $4 / 3$. The values of $\Gamma_{1}$ for $P_{\text {rad }}=0$ at lower $\tau_{\text {Ross }}$ values do not deviate much from the value at $\tau_{\text {Ross }}=2 / 3$. This may indicate that the values found at small $\tau_{\text {Ross }}$ values largely describe the whole atmosphere from $\tau_{\text {Ross }}=2 / 3$ to at least $\tau_{\text {Ross }}=.03$. Around $\log T_{\text {eff }} \approx 3.88$, the difference using $P_{\text {rad }}$ or NLTE is indicated by a much smaller $T_{\text {eff }}$ interval in which $\Gamma_{1}$ shows larger changes. Here a smaller area of more intense instability is indicated. In another $\operatorname{dip}$ at $\log T_{\text {eff }} \approx 4.1$ it is to be expected that $\Gamma_{1}$ for NLTE will decrease significantly below $4 / 3$ at higher $\tau_{\text {Ross }}$-values, going inwards into the stellar atmosphere. The wind starts at lower temperatures of about $\log T_{\text {eff }} \approx 3.8$.

In the graphs, the dynamical-stability-value of $\Gamma_{1}=4 / 3$ is indicated by a horizontal line. Points below that line indicate an "unstable" region, i.e. where mass movements do not oscillate about some stable point but might break up (cf. Kippenhahn \& Weigert 1994).

A comparison of Fig. 1 (Sect. 2) and Fig. 14 shows that the indicated areas of possible instability of $\Gamma_{1}$ seem to coincide with the outer edge of the yellow evolutionary void in the HR-diagram and that there are two smaller regions within the general area of the void, corresponding to $\mathrm{H}$-ionization, and to He-ionization, respectively, that appear to show instability. We will tentatively call these the first yellow void (or yellow void I in short) and the second yellow void (or yellow void II), respectively.

We also note that the red limit of the "total" yellow void also corresponds to a major change in the convective regime, i.e. the convective velocities reach sonic values, cf. i.e. Fig. 16, Maeder (2009, p. 107). Both the turbulent pressure and acoustic 
flux become high below this limit and give rise to the "De Jager Limit" for which we refer to De Jager (1984) and Maeder (2009, Sect. 5.53, and p. 107).

To summarize the above, evolutionary codes from Meynet et al. (1994) indicate that for the modelled stars, the timescales involved for $T_{\text {eff }}$ to evolve from $5000 \mathrm{~K}$ to $8000 \mathrm{~K}$, are as short as some 10000 years or slightly more. For dynamical models of stellar evolution (i.e. including the acceleration term in the equation of motion), this usually corresponds to shorter timescales (by at least a factor of two) than hydrostatic models, where the $10000 \mathrm{yr}$ value might be an upper bound. It is plausible that the underlying star continues its regular evolutionary processes, but that the atmosphere undergoes episodic mass-loss (for example in the earlier history of HR 8752), causing from time to time the star to produce a pseudo photosphere with a different temperature from that of a stable atmosphere, i.e. a lower temperature, because more mass surrounds the star in a circumstellar envelope than in a stable situation. When the pseudo photosphere disperses, the effective temperature increases again (to a maximum temperature that is associated with the evolutionary scenario), until a combination of the critical atmospheric stability parameters again results in a condition of instability in the atmosphere, after which another episode of mass-loss may occur. These processes are expected to continue until the balance of the "atmospheric" accelerations results in a slightly positive effective gravity $g_{\text {eff }}$, allowing the atmosphere to regain stability. It is expected (Nieuwenhuijzen \& De Jager 1995) that in the continuation of its evolution the star will become increasingly stable, until it arrives in the second area of instability, where again stellar instability and/or severe mass-loss may occur, before continuing on its further (possibly complex) evolutionary path (cf. Stothers \& Chin 1995).

\subsection{Evolutionary considerations, the case for $\Gamma_{1}$}

We wish to compare actual evolutionary models of 25-60 $M_{\odot}$ against values of $\Gamma_{1}$ varying over the HR-diagram for a luminosity range in the case of the hypergiants of $\log \left(L / L_{\odot}\right)=5.2-5.8$.

To find the variations in $\Gamma_{1}$, we apply the same procedure as for the specific stellar model to the whole upper part of the HR-diagram by making use of statistical data on mass and massloss to obtain a grid over the HR-diagram of possible stellar evolution data. The mass-loss data is taken from our compilation of rates of mass-loss (De Jager et al. 1988). The stellar mass $M$ at any point in the evolutionary tracks is found from the results of evolutionary calculations by Maeder \& Meynet (1987, 1988) with the aid of a computer program described by us (Nieuwenhuijzen \& De Jager 1990). The code for $\Gamma_{1}$ is taken from Lobel et al. (1992) and Lobel (2001).

In Figs. 17 and 18 we present the contour diagrams for the weighted mean $\left\langle\Gamma_{1}\right\rangle$ as given by Eq. (1) between $\tau_{\text {Ross }}=2 / 3$ and $\tau_{\text {Ross }}=0.03$ for tracks 3 and 4 , respectively. Since the LTE $P_{\text {rad }}=0$ calculations seem to give the clearest indications of the position of the instability regions, we restrict the display to these values. Contour levels are indicated for $\left\langle\Gamma_{1}\right\rangle=1.10-1.40$ to bracket the "edge" of the stability with $\left\langle\Gamma_{1}\right\rangle=4 / 3$. Although we are unable to distinguish a "trigger" point for instability, we do indicate the general region where instability can be expected.

It is interesting to see the marked differences between the evolutionary tracks 3 and 4, especially when we project examples of the models that have also been used in Fig. 1 for ZAMS masses of 25,40 , and 60 solar masses onto the background grid with contour lines for $\left\langle\Gamma_{1}\right\rangle$. We note that in Fig. 18 for model m025e020 the backward loop described in Fig. 1

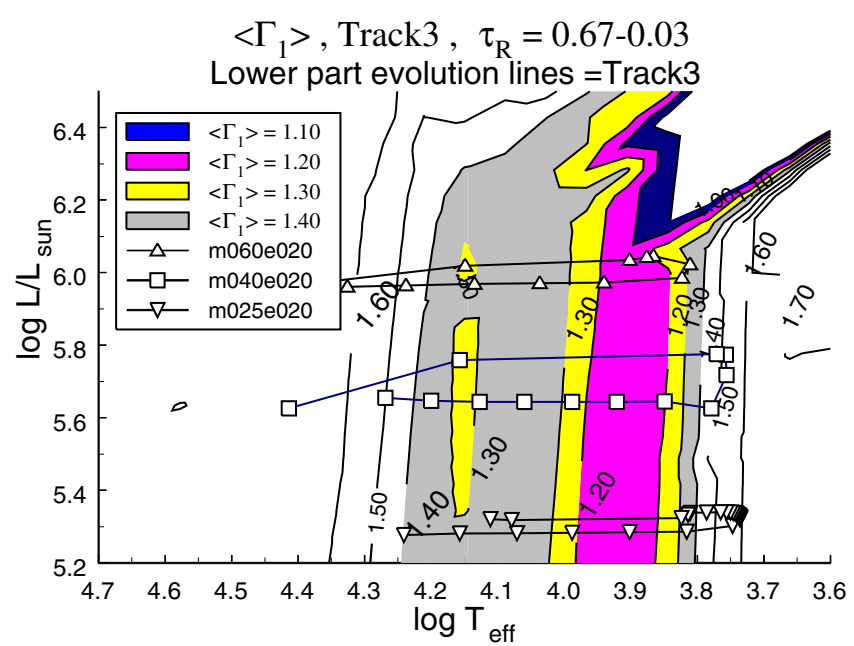

Fig. 17. Evolutionary model tracks for ZAMS masses of 25, 40, and 60 solar masses (Meynet et al. 1994) are superimposed on a background grid with values of $\Gamma_{1}$, as described in the text. $\Gamma_{1}$ is given for the full calculation, no radiation, and following Lobel et al. (1992) and Lobel (2001). The graph should be considered with the lower parts of the evolutionary tracks (track 3 ).

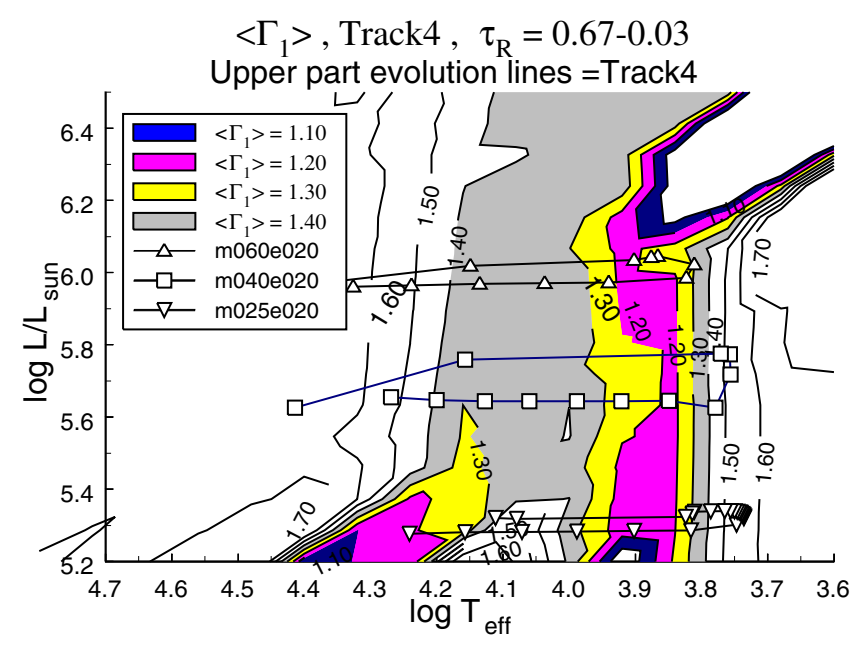

Fig. 18. As Fig. 17, but now for track 4. The graph should be considered with the upper parts of the evolutionary tracks (track 4).

of Sect. 1 is not separately indicated. The models are those of Meynet et al. (1994) with mass-loss twice the value of the massloss given by De Jager et al. (1988). There is one point of caution: the masses used to determine the background grid for $\Gamma_{1}$ are those of Maeder \& Meynet $(1987,1988)$, while the $\Gamma_{1}$ for the evolution traces is based on the masses of the newer Meynet et al. (1994) models. Although details might differ, the general overview is expected to reasonably show the influence of $\left\langle\Gamma_{1}\right\rangle$ over the specified part of the HR-diagram.

With a total pressure $P_{\mathrm{t}}=P_{\mathrm{gas}}+P_{\mathrm{rad}}, \rho_{\mathrm{R}}$ the density of the outermost atmospheric layer, and $\rho_{*}$ the density at the base of the stellar envelope, the weighted mean $\left\langle\Gamma_{1}\right\rangle$, according to Lobel (2001, Eq. (68), and using his notation for the integration limits)

$$
\left\langle\Gamma_{1}\right\rangle=\int_{\rho_{\mathrm{R}}}^{\rho_{*}} \rho \Gamma_{1}\left(P_{\mathrm{t}} / \rho\right) \mathrm{d} \ln \rho / \int_{\rho_{\mathrm{R}}}^{\rho_{*}} \rho\left(P_{\mathrm{t}} / \rho\right) \operatorname{dln} \rho
$$

is an average value over the stellar mass with a weighting that depends on the density of internal energy (e.g. Maeder 2009). In our case, we use $\rho_{R}=\rho_{\tau_{\text {Ross }}=0.03}$ and $\rho_{*}=\rho_{\tau_{\text {Ross }}=2 / 3}$ as 
integration limits. We approximate the integral by the weighted mean of $\Gamma_{1}$ of the two given values of $\tau_{\text {Ross }}$. This value indicates where part of the atmosphere may become instable, but does not give us a number that indicates instability. We do not enter here into a discussion of all the stabilizing and destabilizing effects that occur in the stellar atmospheres, such as those mentioned in Maeder (2009, Eq. (15.70), and Fig. 15.4), but only note that the regions indicated by the $\left\langle\Gamma_{1}\right\rangle$ depressions are uncertain regions in which unstable actions might change atmospheric structures and create regions of excess energy that can be used after a smaller trigger has been created to possibly create large changes in the atmospheric structure.

\subsection{Evolutionary tracks 3 and 4}

While the general contours in Figs. 17 and 18 differ somewhat for tracks 3 and 4 , the values for the $\Gamma_{1}$ parameter are much the same in the corresponding "instability" regions.

We now consider the indicated models for the given ZAMS masses and initiate the evolutionary track 3 in a redward direction (losing mass without encountering instabilities, see above) and going through the "mirror" point, changing direction to blueward at track 4 (where extra mass is lost while encountering instabilities), until some high temperature where we stop. We comment only on model m025e020, although for the other models analogous comments can be made. In Fig. 17 (for track 3), the $25 M_{\odot}$ model starts around $\log T=4.25$, glides along its path, crosses $\log T=4.0,3.9,3.8$, arrives at the "mirror point" at $\log T=3.75$, remains there for some time, and thereafter starts on its blueward track 4, for which we now move to the appropriate Fig. 18. Here the star evolves in smaller (computational) temperature steps $\log T=3.8,3.81,3.82 \ldots$ In the meantime, $\left\langle\Gamma_{1}\right\rangle$ has fallen from $\left\langle\Gamma_{1}\right\rangle=1.60$, to $1.50,1.40$, and 1.30, approaching 1.20. There is then a jump in the computations to higher temperatures.

The 40- and 60-solar-mass models show the same general behaviour. Figure 18 (track 4) shows that whole jumps can occur in the models, thus the star moves through the first yellow evolutionary void (H-ionization), passes onto temporary stability and then passes through the second yellow evolutionary void (He-ionization). Models with higher ZAMS masses (e.g. m080e020) do not extend to such low temperatures but remain at temperatures higher than $\log T \approx 4.1,4.2$. These models have not been included in Figs. 17 and 18.

Although the differences in $\Gamma_{1}$ between tracks 3 and 4 do not seem to be large, the difference in mass is large: the 40 solarmass (ZAMS) star at the start of track 4 has about 32 solar masses, while the instabilities take place at about 21-20 solar masses (cf. Fig. 21). The 25 solar-mass star has a similar behaviour with lower masses of 22 and 13-11 solar masses, respectively (cf. Fig. 22). During the evolution the different masses may well give rise to different structures of the stellar atmosphere for tracks 3 and 4 . In the course of this paper, we estimate actual- and ZAMS masses for HR 8752 in Sect. 5.

We conclude that the determination of low $\left\langle\Gamma_{1}\right\rangle$ values by itself is an insufficient explanation of the "instability" that we discuss here, but that the combination of low $\left\langle\Gamma_{1}\right\rangle$ and appropriate low values of mass may lead to the start of such an "instability" process. The conclusion that not only the adiabatic exponent $\Gamma_{1}$ is important but also the mass is quite interesting, because the layers below the formal photosphere also play a role in the instability. However, apart from the aforementioned data from the evolutionary models, we study no data range within which these phenomena do exist. We also refer to the influence of various timescales in Sect. 5.

What happens when a star moves through an H-ionization region where $\left\langle\Gamma_{1}\right\rangle$ gets smaller than 4/3? In Fig. 14, we present the evolution of one of the Meynet models to reach a value of $\Gamma_{1}$ at $\tau_{\text {Ross }}=0.666$, which is also representative of most outer layers, that lies below the critical value of $\left\langle\Gamma_{1}\right\rangle=4 / 3$. Here, the time increases from right to left. The model shows the blueward evolution after the red/blue "mirror" point, where the star goes to higher temperatures. The value of $\Gamma_{1}$ in the atmosphere reaches the critical limit of instability at around $\log T_{\text {eff }}=3.78$, but perhaps only locally in a small region. It would be interesting to know the fractional mass $(\Delta M / M)$ (cf. Sects. 5.5, 6.1, 6.3) when smaller or larger events arise. Perhaps a large part of the whole star with $(\Delta M / M) \approx 1$ may become critical and result in a significant event such as the "1973" event. We can discern in the acceleration graph (Fig. 16) an "instability" (a peak) in the wind acceleration occurring around $\log T_{\text {eff }}=3.80$ which may indicate some form of "instability" over a large part of the atmosphere as given in Fig. 23 as step I or II.

\subsection{The "Geyser model"}

From other data, we know that HR 8752 underwent an episode in about 1973 during which it ejected a large amount of mass, had a large diameter, and a low temperature. We may speculate about why massive stars eject substantial amounts of mass when instability is met. This may be related to the thermal timescale $t_{\text {therm }}$ in supergiants being shorter than the dynamical timescale $t_{\mathrm{dyn}}$, while the opposite is true in intermediate and low mass stars. If $t_{\text {therm }}<t_{\text {dyn }}$, this means that after the dynamical ejection of some of the outer layers (e.g. when $\Gamma_{1}<4 / 3$ ), any thermal instability associated with the ionisation peak, or either the supraEddington or supra-De Jager layers, will be able to move very rapidly inwards to contribute to the further ejection of matter from deeper layers, causing large eruptions. This is the basic idea of the so-called "Geyser model" (Maeder 1989, 1991).

Lobel et al. (2003) proposed a mechanism to explain the recurrent outbursts in $\rho$ Cas due to an avalanche of hydrogen recombination releasing the ionization energy and driving a global eruption of its atmosphere observed in 2000-2001. The hydrogen-recombination mechanism predicts an outburst timescale of 221 days over which the radius $R$ of $\rho$ Cas temporarily increased by a factor of $\approx 2.5$.

For HR 8752, the immediate result of the 1973 event was that the star contracted, the effective temperature rose and the star ultimately changed into a hotter star with a smaller radius. The amplitude of the $T_{\text {eff }}$ increase is directly related to the amount of mass ejected in the eruption, as suggested by simulations of sudden mass ejections (Maeder 1989, 1991). After that, the star resumes its secular evolution and recovers the $T_{\text {eff }}$ it had just before the eruption, with a timescale that depends on its mass. However, the models indicate (cf. Sect. 5.7, Fig. 23) that the star resumes its secular evolution with a small step towards higher evolutionary temperatures. It may then again either meet the instability and experience another eruption or be part of and start a more complex cycle. This eruptive cycle may only stop at the time of the supernova explosion, unless the totality of the hydrogen-rich layers is ejected before.

\subsection{Conclusions of this section}

A significant observation arises from Fig. 14, viz. that the instability region that we originally called the yellow evolutionary 
void, actually consists of two separate instability regions, one for $3.8<\log T_{\text {eff }}<3.95$ and another at higher temperatures $4.05<\log T_{\text {eff }}<4.15$, which we call yellow evolutionary void I and II, or yellow void I and II, respectively. We also found that the void is traversed in a cosmologically short time, which explains why practically no stars are found in that part of the HR-diagram.

\section{HR 8752: (Hyper-)giant steps in evolution?}

We plot stellar parameters as a function of time: temperature $T$, luminosity $L$, radius $R$, and mass $M$, and compare dynamical and thermal timescales with observed variations, and finally compare wind-driven mass-loss from evolution models with observed high mass-loss caused by cycles possibly of the $\Gamma_{1}$-Geyser process. Is this last process more efficient in losing mass than the classical wind-driven mass-loss?

To help us ascertain where the observed hypergiant may then be situated on its evolutionary path we introduce the concept of evolutionary temperature as opposed to that of the atmospheric temperature.

\subsection{Atmospheric and evolutionary temperatures}

We display $T_{\text {eff }}$ in Fig. 10 (cf. Sect. 3) as a function of time. We now define the evolutionary temperature (which we call $T_{\text {Blue }}$ ) as the highest observed temperature that would exist in bluewards evolving hypergiants which is identical to the temperature of the evolution model and unaffected by any changes to lower temperatures in the atmosphere of the evolving star. A well-determined $T_{\text {Blue }}$ can help us to determine where the star is situated in the model scenario. For hypergiants, the atmospheric and evolutionary temperatures may be quite different, and we should not use the varying atmospheric temperatures to find masses from selected tracks in evolution models.

Figure 10 shows that while a long period of nearly constant temperature lies around $\log T_{\text {eff }}=3.7$, there is a cluster of points around $\log T_{\text {eff }}=3.73$ and finally at the end of the record a cluster of points around $\log T_{\text {eff }}=3.90$. We equate this maximum temperature to the evolutionary temperature of $\log T_{\text {Blue }}=3.90$ for HR 8752 as a best (or minimum) estimate.

\subsection{Luminosity $L$}

The value $\log L$ is shown in Fig. 19 as a function of time using $\log L=0.4\left(M_{\mathrm{bol}, \odot}-M_{\text {bol,star }}\right)$ with $M_{\text {bol, } \odot}=4.64$ (Schmidt-Kaler 1982, Sect. 4.1.5.1). In the figure, the luminosity increases from $\log \left(L / L_{\odot}\right) \approx 5.4$, to $\log \left(L / L_{\odot}\right)=5.6$, and then reduces to $\log \left(L / L_{\odot}\right) \approx 5.3$. There seems to be an overshoot, after which the luminosity seems to stabilize at $\approx \log \left(L / L_{\odot}\right) \approx 5.33$. The temporary overluminosity may result from the thermal radiation of internal energy that comes free in that part of the process (cf. thermal timescale below). During a more stable part of the process, when no large thermal timescale variations are visible, the luminosity is expected to be equal to the classical luminosity value. The "mean" reference value (cf. Sect. 3, step 4) of $\log \left(L / L_{\odot}\right)=5.384 \pm .296$ is expected to be a classical value of luminosity and neither over- nor under-luminous, and to be valid for a short time before the episode of $\approx 1965-1980$. We designate this point with the code L1 for later use. If the overshoot were real, it could mean that the values at the end of the observational record define a new "stable" point of luminosity after the "event" around the year 1973. We designate this point $\log L / L_{\text {sun }}$ for period 1942-1996

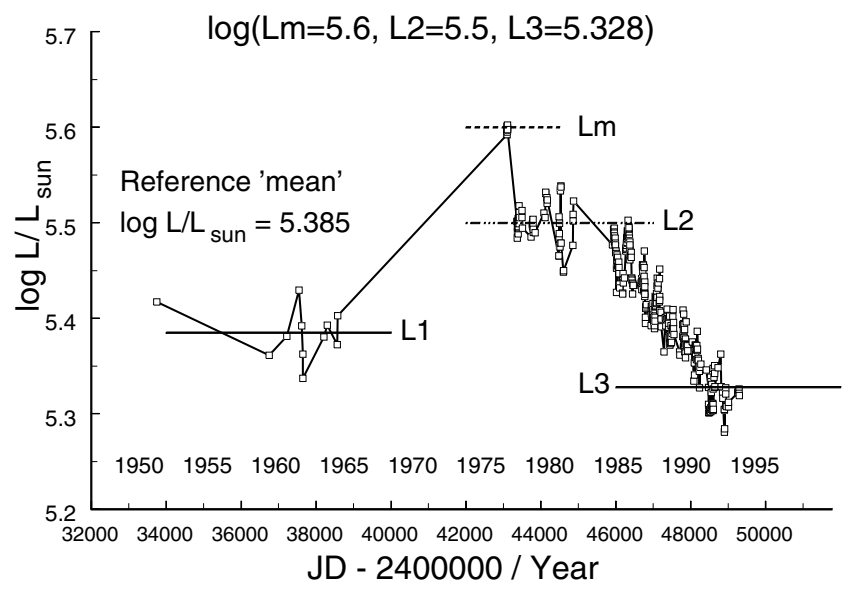

Fig. 19. $\log \left(L / L_{\odot}\right)$ as a function of time, timeframe 1942-1996.

$\log R / R_{\text {sun }}$ for period 1942-1996

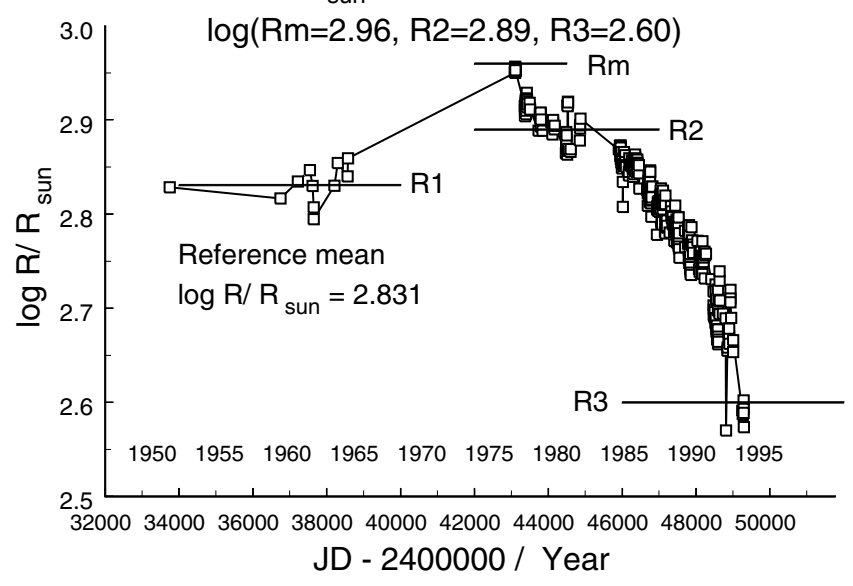

Fig. 20. $\log \left(R / R_{\odot}\right)$ as a function of time in the timeframe 1942-1996.

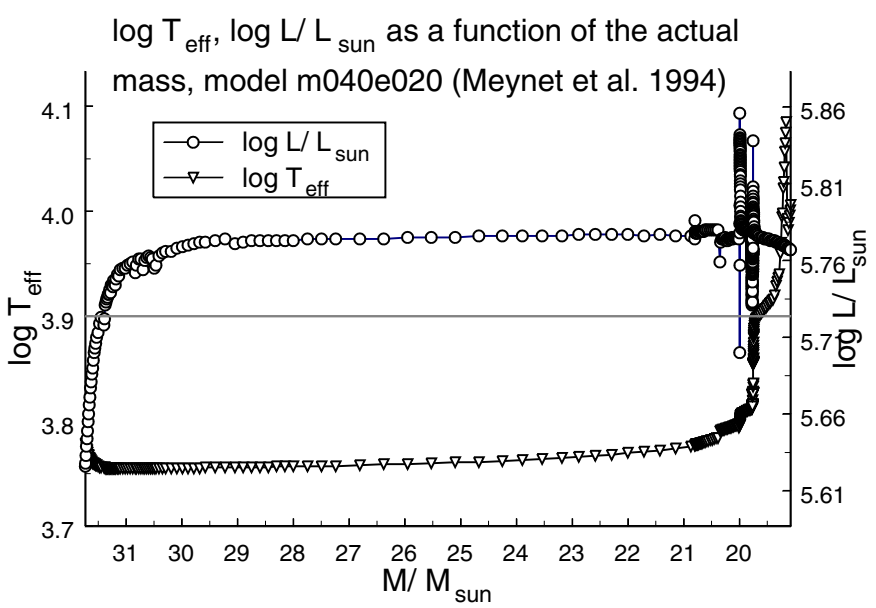

Fig. 21. $\log T_{\text {eff }}$ and $\log L / L_{\odot}$ as a function of the actual mass for model m040e020 (Meynet et al. 1994), track4, with a horizontal line at $\log T_{\text {eff }}=3.90$.

with the code L3 for later comparison with point L1. We note however the variation around the year 1980, which we designate L2. This point might signal the end of the shell ejection. The maximum observed bolometric luminosity $\mathrm{Lm}$ is indicated in Figs. 19 and 24. 
$\log T_{\text {eff, }}, \log L / L_{\text {sun }}$ as a function of the actual

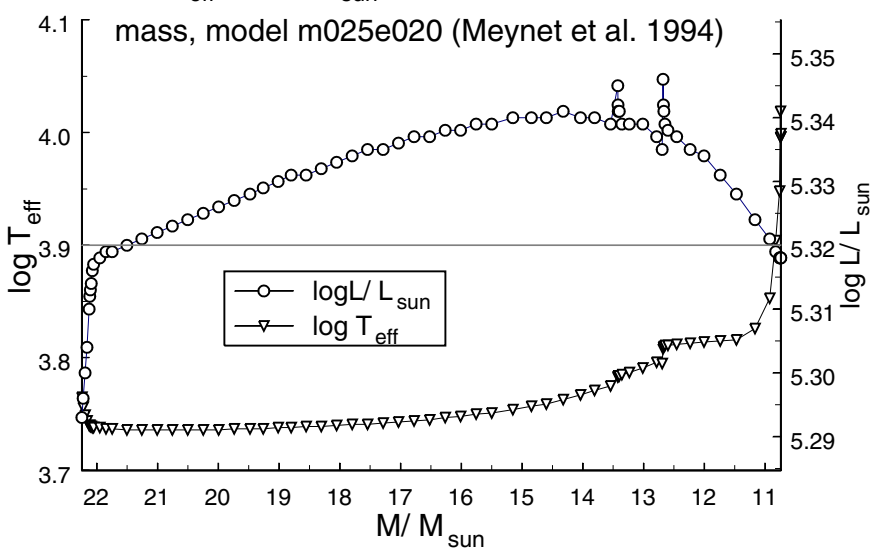

Fig. 22. As Fig. 21 but now for model m025e020 (Meynet et al. 1994), track4, with again a horizontal $\operatorname{line}$ at $\log T_{\text {eff }}=3.90$.

$\log T_{\text {eff }}, \log \mathrm{L} / \mathrm{L}_{\text {sun }}$ as a function of the actual

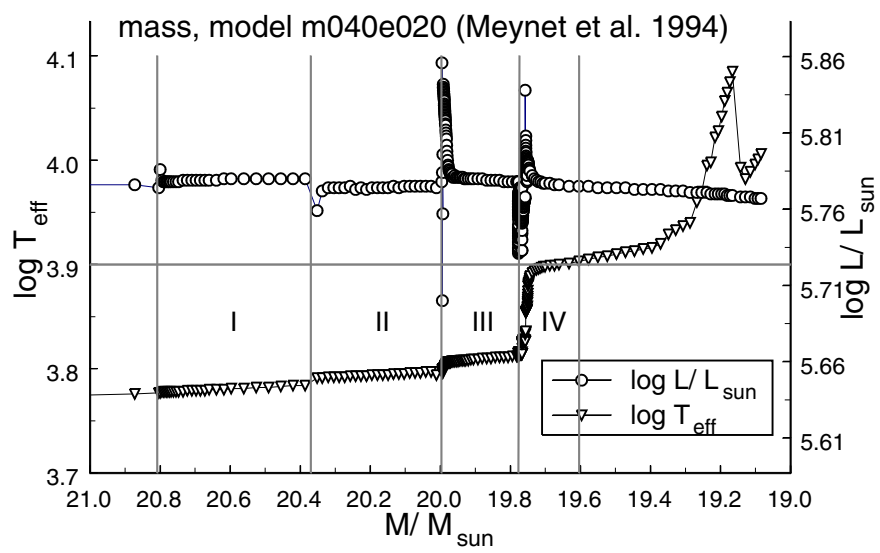

Fig. 23. $\log T_{\text {eff }}$ and $\log L / L_{\odot}$ as a function of the actual mass for model m040e020 (Meynet et al. 1994), in greater detail than Fig. 21.

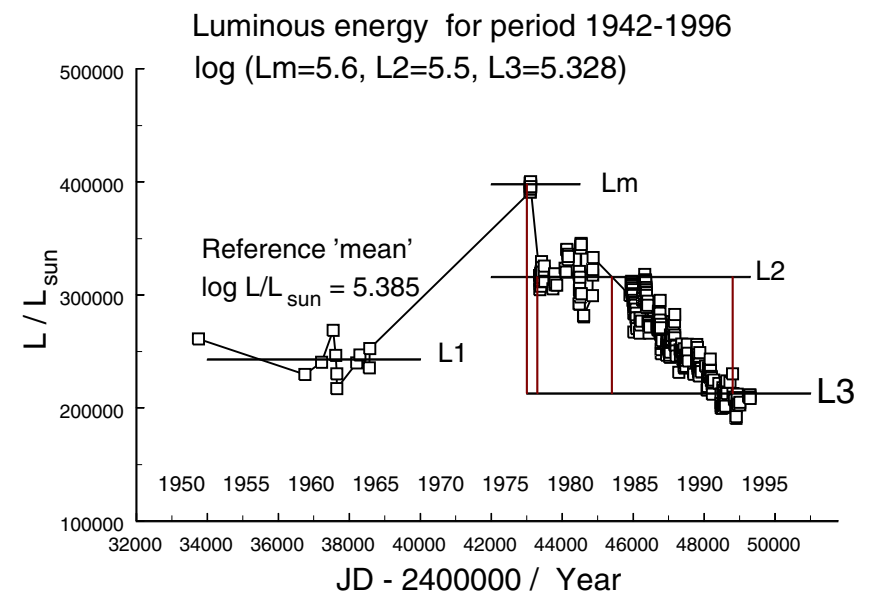

Fig. 24. Linear plot of luminosity of HR 8752 in the timeframe 1942-1996, with integration lines.

The conclusion that the total luminosity varies so widely, if true, is a major result, that in the future would require a physical explanation. It remains unclear, however, which changes in the composition and opacities occur in the outer layers (cf. comments in Sect. 3, step 4 for two possible interpretation routes).
Table 7. Actual mass, luminosity, and calculation step for the indicated model and $\log T_{\text {Blue }}=3.90$.

\begin{tabular}{lccccc}
\hline \hline$M_{\text {ZAMS }}$ & $\log T_{\text {Blue }}$ & $M_{\text {actual }}$ & $\log L / L_{\odot}$ & Model & Step \\
\hline 40 & 3.903 & 19.60 & 5.775 & m040e 020 & $\# 2591$ \\
25 & 3.904 & 10.83 & 5.319 & m025e020 & $\# 1291$ \\
\hline
\end{tabular}

\subsection{Radius $R$}

The value of $\log R$ is shown in Fig. 20 as a function of time using $L=4 \pi R^{2} \sigma T_{\text {eff }}^{4}$ written as $\log R / R_{\odot}=-0.20 M_{\text {bol }}-2 \log T_{\text {eff }}+$ 8.47 (Schmidt-Kaler 1982, Sect. 4.1.5.2). From a quasiequilibrium situation, with deviations of \pm 0.02 , the radius appears to increase slowly from $\log \left(R / R_{\odot}\right)=2.82$ (in 1950-1965) to $\log \left(R / R_{\odot}\right) \approx 2.95$ after the episode $(1965-1980)$, and then diminish relatively rapidly to $\log \left(R / R_{\odot}\right) \approx 2.59$. The total visible change in the radius itself is by more than a factor of two. Here a small overshoot (cf. Luminosity above) to a more stable situation is also apparent. The numbering of the R-codes indicate the same observing points as the L-codes.

We presume that the time constants in the atmospheric processes allow the radius $R$ to follow the changes in $T_{\text {eff }}$ and $L$, so that the overshoot seems to be a real phenomenon.

\subsection{Mass $M$}

The stellar mass $M$ is the basic stellar parameter. We try to determine the actual mass of HR 8752 using the mass-luminosity relation developed below together with a value of the evolutionary temperature $T_{\text {Blue }}$. The relation is valid only when the luminosity values are stable, so that with the variations in luminosity observed in HR 8752, we can only get apparent masses, but we hope to find points where the luminosity can be taken as a measure of a stable situation, leading to an assessment of real actual mass.

We do not present a graph of the apparent mass, but only define two observational points at which the luminosity is $\approx$ stable, and that can help us to estimate the amount of mass lost in the "1973" event of photospheric ejection.

From the Meynet et al. 1994 evolutionary models m040e020 and $\mathrm{m} 025 \mathrm{e} 020$ (cf. Sect. 4), we derive the following limited mass-luminosity relation. Figures 21 and 22 show the temperature and luminosity as a function of (diminishing) mass for these models with ZAMS masses of 40 and $25 M_{\odot}$. We limit ourselves to the red supergiant evolution going bluewards from the "mirror" point (track 4). For our analysis of HR 8752, we limit ourselves to the temperature $\log T_{\text {Blue }}=3.90$.

Where the horizontal line at $\log T_{\text {eff }}=3.90$ in Figs. 21 and 22 crosses the temperature plot, the axis indicates the value of the actual mass for both the 40 solar mass and $25 M_{\odot}$ ZAMS models. Where the vertical line through this mass point crosses the luminosity graph, we find that the luminosity agrees with the given temperature and derived mass. The respective values of the 40 solar mass and $25 M_{\odot}$ ZAMS models are collected in Table 7, its third and fourth columns.

With these data, we construct a mass-luminosity relation for a hypergiant in track 4 limited to a given evolution temperature, the coefficients of which are given in Table 8 for mass as a function of (observed) luminosity. The relation has been derived for hypergiants evolving along track 4 with ZAMS masses in the range $25-40 M_{\odot}$. It is this relation that we use to derive actual masses and also the original ZAMS mass of the hypergiant. 
Table 8. Interpolation coefficients for "actual" and ZAMS masses $\log M / M_{\odot}=a+b \times\left(\log L / L_{\odot}\right)$ for $\log T_{\text {Blue }}=3.90$.

\begin{tabular}{lcc}
\hline \hline Coeff & For $\log M_{\text {actual }} / M_{\odot}$ & For $\log M_{\text {ZAMS }} / M_{\odot}$ \\
\hline $\mathrm{a}$ & -1.9373 & -0.9628 \\
$\mathrm{~b}$ & 0.5587 & 0.4437 \\
\hline
\end{tabular}

Table 9. Basic stellar parameters for special points in time (values and logarithmic values).

\begin{tabular}{lccccc}
\hline \hline Point & $\log L$ & $\log R$ & $\log M$ & $t_{\text {dyn }}$ & $t_{\text {therm }}$ \\
\hline units & $L_{\odot}$ & $R_{\odot}$ & $M_{\odot}$ & $\mathrm{d}$ & $\mathrm{yr}$ \\
\hline L1 & 5.385 & 2.831 & 1.07 & 93 & 27 \\
Lm & 5.6 & 2.96 & $\ldots$ & 146 & 15.7 \\
L2 & 5.5 & 2.89 & $\ldots$ & $\ldots$ & $\ldots$ \\
L3 & 5.328 & 2.60 & 1.033 & 43 & 55.6 \\
\hline
\end{tabular}

Table 8 contains the interpolation coefficients to find the mass and original ZAMS mass from the observed luminosity values for the indicated temperature. We assume that the luminosity is observed over a period of no obvious over- or underluminosity of the star.

The (observed) reference luminosity $\left(\log L / L_{\odot}=5.384\right)$ for HR 8752 is close to that of the $25 M_{\odot}$ ZAMS model, hence the actual mass associated with the indicated temperature is estimated to be almost $10.8 M_{\odot}$. Using the derived mass-luminosity relation and assuming $\log T_{\text {Blue }}=3.9$, we find for the aforementioned points L1 and L3 $M_{\text {actual }}(L 1)=11.78$, $M_{\text {actual }}(L 3)=10.96 \pm 0.09$ (relative to L1) and $M_{\text {ZAMS }}(L 1)=$ $26.67, M_{\text {ZAMS }}(L 3)=25.23$, in solar units. Owing to the uncertainties in $M_{\text {bol }}$ (cf. Sect. 3), the absolute logarithmic error in the actual mass is 0.168 (a factor of 1.471) and for the ZAMS mass 0.133 (a factor of 1.36).

We conclude that the most consistent estimate of the mass $M$ corresponding to the high temperature of $\log T_{\text {Blue }}=3.90$ for point L3 is $M_{\text {actual }}=10.96 M_{\odot}$ with given accuracies, and for the ZAMS mass is $M_{\mathrm{ZAMS}}=25.23 M_{\odot}$ with its given accuracies. The other estimate L1 has a known atmospheric- but an as yet unknown evolutionary temperature. The $25 M_{\odot}$ evolutionary model with $\log T_{\text {Blue }}=3.90$ can effectively be used to approximately describe the position of HR 8752 during its (proposed) evolution.

\subsection{Dynamic and thermal timescales $t_{d y n}$ and $t_{\text {therm }}$}

We investigate the timescales that appear in the observations of HR 8752 to determine the kind of processes that might occur and lead to the indicated instabilities (cf. Sect. 3).

The dynamical timescale gives an indication of the time period during which a (dynamically stable) star reacts following a slight perturbation of the hydrostatic equilibrium. These values change with the atmospheric variation in historical time: interesting points for HR 8752 are the mean $(\approx$ stable) time (Sect. 3, step 2) given as point L1, the highest luminosity given as point Lm, and the end of the observation period, with slightly more than the lowest luminosity given as point L3. In the following definitions, we consider the mass derived at point L1: $\log M=1.07$.

For $t_{\mathrm{dyn}}$, we have $t_{\mathrm{dyn}} \approx\left(R^{3} /(\mathrm{G} M)\right)^{1 / 2}$ (Maeder 2009, Eq. (1.28); Kippenhahn \& Weigert 1994, Eq. (2.19): $t_{\mathrm{hydr}}=t_{\mathrm{dyn}}$ ). We give values for the aforementioned points of HR 8752 in Table 9. To find the dynamical timescale corresponding to a shell mass $\Delta M$ of thickness $\Delta R$, we multiply the given numbers with the factor $(\Delta R / R)^{1 / 2}$ (Maeder 1992). For the Sun, $t_{\mathrm{dyn}} \approx 27 \mathrm{~min}$.

The thermal timescale $t_{\text {therm }}$ gives an indication of the time needed to radiate internal energy, and has the same order of magnitude as the Kelvin-Helmholtz timescale during which a star can produce a luminosity $L$ from the gravitational energy. For $t_{\text {therm }}$, we have $t_{\text {therm }} \approx \mathrm{G} M^{2} /(R L)$ (Maeder 2009, Eq. (3.50); Kippenhahn \& Weigert 1994, Eq. (3.19): $t_{\text {therm }}=t_{\mathrm{KH}}$ ). We give values for the aforementioned points of HR 8752 in Table 9. These times indicate that any process acts over the whole star, while when considering smaller mass fractions we multiply the given numbers by the mass fraction $(\Delta M / M)$. For the Sun, $t_{\text {therm }} \approx 1.6 \times 10^{7}$ years.

In the Geyser model, the equality of the two timescales applies to the thin superficial layers that are ejected in the outburst, as mentioned in Sect. 4 of the Maeder (1992) paper. In this case, the thermal timescale has to be applied to a mass fraction $(\Delta M / M)$ of the total stellar mass (see formula on page 141 of Maeder 1992), where the mass fraction is generally very small and estimates in the above paper ranged from 0.01 to 0.0001 . The dynamical timescale applies to $\Delta R$, which is of the same order of magnitude in yellow supergiants as $R$. Thus, if these are taken into account, things are globally consistent with the comment about the "Geyser model" at the end of Sect. 4. For more information, we refer to Maeder (1992).

In Sect. 6, we derive values of the mass fraction $(\Delta M / M)$ that permit us to compare theoretical timescales with observations.

\subsection{Comparing $t_{d y n}$ and $t_{\text {therm }}$ with the observed variations}

It is interesting to compare these times with the observations (cf. Table 6 and the Conclusions in Sect. 3) which display evidence of different timescales for the variations. We use the same identifiers and present the four following types:

1) A short timescale $\approx 200-300$ days, as described in earlier papers (Nieuwenhuijzen \& De Jager 2000; Percy \& Zsoldos 1992). This seems common in very heavy stars (cf. van Leeuwen et al. 1998). Such short times represent the $d y$ namical timescales, and are responsible for the variability of the star by about $0.2 \mathrm{mag}$.

2) A medium timescale $\approx 2-5$ years, which relates to the movements described earlier in this paper (Sect. 3), cf. Figs. 10 and 11 . We propose that they are of the same type as the "events" described for the hypergiant $\rho$ Cas by Lobel et al. (2003) for 1945-1947 $(V \approx 5.6-7.0), 1985-1987(V \approx$ 4.4-5.2), and 1991-2001 ( $V \approx 4.1-5.4)$. We also note that Zsoldos \& Percy (1991) studied $\rho$ Cas in the period 1963-1989 finding short timescale variations, as in 1) above with $V \approx 4.175-4.692$ and a deep minimum in 1986 consistent with Lobel et al. (loc. cit.). These medium timescales seem to be similar to the thermal timescales for a fraction of the $\operatorname{star}(\Delta M / M)$ with $(\Delta M / M) \approx 0.01-0.1$ to obtain the appropriate time found for the values of $(\Delta M / M)=1$ given above.

We note that the "events" for the hypergiant $\rho$ Cas (Lobel et al. 2003), in the periods 1985-1987 and 1991-2001 and Zsoldos \& Percy (1991) in the period 1963-1989 seem to be of type 2. However, the "event" for the hypergiant $\rho$ Cas (Lobel et al. 2003) in the period 1945-1947 with $V \approx$ 5.6-7.0 seems to be of type 3 below.

3) A shell ejection "event" around 1973 with a timescale of $\approx 20$ years, which may make part of timescale type 4 below. 
4) A long time variation of longer than 100 years. This long timescale involves a complex interplay of a number of timescales, cf. the "Geyser effect" in Sect. 4, and comments in Maeder (1989, 1992). The longer shell ejection/ recovery times of types 3 and 4 are probably indicative of a thermal timescale for a large fraction of the star $(\Delta M / M)$ with $(\Delta M / M) \approx 1$. While there is a long-term (>100 year) scenario involving either a large part or the whole star, where shorter time disturbances may point to "instabilities" in a small part of the atmosphere.

We note that for all timescales taking $(\Delta M / M)=1$ refers to the whole star and the dynamical time is always shorter than the thermal timescales. In all stars and low enough $(\Delta M / M)$, the thermal timescale can be shorter than the dynamical timescale in view of their respective dependence on $(\Delta M / M)$.

The main question is whether the fractional mass $(\Delta M / M)$ where the equality is reached is below the layer where $\mathrm{H}$ starts to be fully ionized. If yes, the Geyser model may clearly work since a large amount of ionization energy may be released when powering the outburst. In Sect. 6, we discuss the energy considerations that may allow us to estimate the shell mass $(\Delta M / M)$ itself.

In overview, we conclude that the variations of type 1 point to dynamical instabilities that are well studied and by themselves do not appear to lead to the variations $2-4$.

In view of the temperature and luminosity areas addressed in Sect. 4 that are responsive to instabilities of the $\Gamma_{1}$ type, we now consider mass lost in tracks 4 of the aforementioned evolutionary models and compare those with the results of the complex $\Gamma_{1}$-Geyser mechanisms introduced in Sect. 4.

\subsection{Mass lost in evolutionary models}

The evolutionary data in Figs. 21 and 22 for m040e020 and m025e020, respectively, for track 4 show the relevant temperature and luminosity values as a function of (diminishing) mass from the "mirror" point to a temperature of at least $\log T_{\mathrm{eff}}=4.0$ with a gradual decrease in mass until the rising temperatures reach the range of $\log T_{\text {eff }} \approx 3.8$. Both models have then lost approximately one third of their mass since the "mirror" point, and then enter into instabilities that rapidly cause the star to lose a few more solar masses before reaching $\log T_{\text {eff }}=3.9$. From the models, it seems that the star then leaves the area of the first yellow evolutionary void, passes through a more quiet region and then enters the second yellow evolutionary void with new instabilities.

The right-hand parts of Figs. 21 and 22 for m040e020 and $\mathrm{m} 025 \mathrm{e} 020$, respectively, show some nearly constant steps of temperature as a function of mass. Some process induces steps of discrete mass-loss of $\approx 0.4$ and $\approx 1 M_{\odot}$, respectively. There is only a small number of discrete steps in mass for both models, which we show in more detail for model m040e020 in Fig. 23.

Figure 23 shows details of Fig. 21 as a function of age for $\mathrm{m} 040 \mathrm{e} 025$. In the figure, we indicate with vertical lines the possible start and end times of the mass-loss-step processes. For the four separate steps, the temperature is in the range $\log T_{\text {Blue }} \approx 3.77-3.90$. For model m025e020, we also find four separate steps in the temperature range $\log T_{\text {Blue }} \approx 3.76-3.90$. In Table 10, we summarize the mass change over this period, the mean mass lost per step in the four steps, and the mean evolution time per step during the four steps.

In both analyses, the fourth step ends at $\log T_{\text {Blue }}=3.90$, which is near to or on the high temperature boundary of yellow evolutionary void I.
Table 10. Mean mass-loss steps and mean time steps for indicated models for track 4 with $\log T_{\text {Blue }}=3.76-3.90$.

\begin{tabular}{lcccc}
\hline \hline Model & Steps & $\begin{array}{c}\text { Change } \\
\text { Mass }\left(M_{\odot}\right)\end{array}$ & $\begin{array}{c}\text { Mean step } \\
\Delta M\left(M_{\odot}\right)\end{array}$ & $\begin{array}{c}\text { Mean step } \\
\text { time }(\mathrm{yr})\end{array}$ \\
\hline $\mathrm{m} 040 \mathrm{e} 020$ & 4 & $20.8-19.6$ & 0.30 & 1470 \\
$\mathrm{~m} 025 \mathrm{e} 020$ & 4 & $14.3-10.8$ & 0.87 & 46000 \\
\hline
\end{tabular}

Viewing this analysis, we make the following observations:

1) Quite generally, the computation of the phases when the star evolves back from the red to the blue is not easy in the sense that the numerical model has difficulty in finding a good fit between the solution for the envelope and the interior.

2) This numerical difficulty probably has a physical cause. It could be due to the envelope encountering some instabilities, as found in our observations of HR 8752. These instabilities would probably be far more accurately followed with a code that did not impose hydrostatic equilibrium and incorporate a more accurate treatment of convection.

3) In this situation, with the present hydrostatic code, we are able to overcome the difficulty by relaxing for a few models the degree of consistency between the envelope and the interior solutions. This may cause short-term variations in the luminosity, effective temperature, and massloss (since this is for very short times, which is not reflected in the variation in the total mass of the star as can be inferred in Fig. 23).

4) In this case, we would not consider these variations in luminosities as a natural outcome of the model, but as an indication that something actually happens in that particular region of the HR diagram. To proceed, we should compute models including the accelerating term in the movement equation and search for what happens using short time steps. For instance, some results with such a code for red supergiants were published by Yoon \& Cantiello (2010). They find pulsation periods deduced from the evolutionary code.

5) The large mass-loss steps in the Stothers \& Chin (2001) models are imposed only at the beginning of the blue loops. Otherwise, evolution along the blue loops proceeds normally in the same way as for the rest of the evolutionary track. The blue loop shown in Fig. 2 of their paper would probably have a total lifetime of $\approx 10^{3} \mathrm{yr}$ unless another episode of enhanced mass-loss created by dynamical instability occurred before the star returned to the region of red supergiants. In real stars, the latter possibility may be quite high, and so the total loop time may actually be irrelevant. A shorter blue loop would also in any case, have a shorter lifetime. This can be easily inferred from Fig. 2.

6) It is impossible to say from Stothers \& Chin's (2001) limited set of models how far blueward a sequence of triggered blue loops along an initial blue loop would push the star. However, the star would certainly maintain its blueness, as their tests have shown.

7) The observations that we have presented above show much higher mean mass-loss rates than the nearly "continuous state" mass-loss of De Jager et al. (1988). Additional processes must be involved to account for these higher rates. When a large amount of mass-loss at the low end of the temperature loop has been introduced, evolutionary codes are then able to trigger and follow the lifetime of the blue loops (cf. Stothers \& Chin 2001). Whether this is also the case when introducing large mass-loss at the high end of the temperature loop is as yet unclear. 
Considering the rate of mass-loss, we now consider some options and indicate limitations:

8) With the concept of $T_{\text {Blue }}$, we consider an evolutionary option with a monotonically increasing temperature where some changes (instabilities) occur with lower atmospheric temperature, considerable mass is lost, there is a temporary increase in the atmospheric luminosity and the maximum attained temperature rises to $T_{\text {Blue }}$. Depending on one's ideas, this could be called either a red loop or a blue loop.

9) The models of Meynet et al. and Stothers \& Chin (short version of consideration 5 above) are described below. The models of Yoon \& Cantiello are very interesting in their procedure, but are outside the range of our observations. The results clearly show that the "classical" mass-loss of De Jager et al. (1988) is insuffient to explain the larger mass-losses indicated below (cf. also Yoon \& Cantiello 2010).

10) Meynet et al. (1994) detected instabilities after an integrated mass-loss of $\approx 1 M_{\odot}$. If the time between instabilities is long this may indicate that greater mass-loss is required to obtain inter-instability-times of $\approx 300$ years. Magnifying Fig. 23 for model m040e020 and looking at the time history, we can see the following. In the time interval 6000-7200 years with respect to a modified JD $=$ JD-4 448000 years, the instability starts with a downward luminosity jump, followed by an upward jump. This behaviour possibly indicates first a period of mass-loss, followed by an "explosion", and seems to mimic our observation graph in Fig. 19.

11) Another interesting point is shown on the right-hand side of Fig. 23, where the luminosity changes very slowly, while the temperature increases, first slowly (stable?), then rapidly towards a maximum of $\log T_{\text {eff }}=4.08$, and falls back quickly to $\log T_{\text {eff }}<3.98$. Could this be interpreted as a jump to the second yellow void, after a time followed by an "instability" leading to temporarily lower temperatures? We also refer to Figs. 14 and 15, which shows the start of the instability coming from the right in the time direction just at the start of the zone where dynamical instability may occur. Is this when the mass-loss must intensify before an "event"? In both examples, the mass loss timing of two times the "classical" value (De Jager et al. 1988) seems to permit the evolutionary code to yield in any case interesting results. We consider the amount of mass-loss as "appropriate", and that possibly also the lower actual mass plays a role in finding an optimum mass-loss to describe observable effects?

12) Yoon \& Cantiello (2010) found scenarios for relatively larger mass-loss rates and found large deviations in evolution for stars at lower temperatures.

13) Stothers \& Chin (2001, Fig. 2) show that by introducing a larger mass-loss for $\log T_{\text {eff }}<3.7$, a fast change to higher temperature (blue loop) is introduced, followed by a slower decay in the temperature on a timescale of $\approx 1000$ years. This could result in a sequence of loops.

14) Maeder (1989, Fig. 2) shows a light curve illustrating the $\Gamma_{1}$-Geyser effect for a $\mathrm{LBV}$, resulting from shell ejections of $1 M_{\odot}$ at various high mass-loss rates. If this mechanism were applicable to yellow hypergiants, it might explain the observations.

15) Combining the above, mass-loss rates of $\approx .01 M_{\odot} \mathrm{yr}^{-1}$ seem to lead to results that can be compared with the observations, but this will have to be verified in more detail. It also seems that when the total sum of mass loss is more than a critical amount, some strong fluctuations (event, instability?) occur with high luminance output followed by a gradual rise in temperature (to $T_{\text {Blue }}$ ?). Can one hypothesize that (part of) the continuous mass loss is collected until such a time that a large instability is created and (part of) the collected mass is ejected in an explosion, while perhaps another part returns to the star (cf. Sect. 6.3)?

16) There is a set of observations of yellow supergiants (hypergiants) in the LMC (Hagen et al. 1981) with estimated mass losses of from $4 \times 10^{-4}$ to $4 \times 10^{-2} M_{\odot} \mathrm{yr}^{-1}$, found with an appropriate value of the inner radius of a circumstellar shell. The mean logarithmic values of $(-) 2.40$ are included in De Jager et al. (1988, Table 1 for NR's 269-273). They were indicated as doubtful values not included in the model calculations. In the context of the above considerations, these values could be consistent with the high mass-loss values needed for the evolutionary modelling.

17) The conclusion of this subsection is given as Table 10, where we see that to enable observed times, the mass loss for model m040e 020 could be a factor 3-4 higher, and for model m025e020 one would think of a factor 100 higher ( -2 in the logarithmic mass loss scale).

\subsection{Observed mass lost (shell ejection)}

The actual mass $M$ and the ZAMS mass of HR 8752 were determined above for the evolutionary temperature $T_{\text {Blue }}$ at two defined instances in time: the indicated instances of observed semistability before and after the "event" around 1973, with indicated luminosities of L1 and L3, using the developed mass-luminosity relation given in Table 8 .

If the luminosities at the designated points were representative of a " $\approx$ stable" atmosphere, in which extra processes have been worked out as far as over- resp under-luminosity are concerned (cf. timescales above), then the application of the derived mass-luminosity relation might give an estimate of the (real) actual mass for points L1 of $M_{\text {actual }}=11.78 M_{\odot}$ and L3 of $M_{\text {actual }}=10.96 \pm 0.09 M_{\odot}$ (relative to L1), with absolute logarithmic errors of 0.168 (factor of 1.471) owing to the uncertainties in $M_{\text {bol }}$ (cf. Sect. 3). We have assumed that to first order the temperature does not change.

The mass lost in the 1973 shell ejection "event" including the 1980 variations is then $11.78-10.96 \pm 0.09=0.82 \pm 0.09 M_{\odot}$ with an absolute logarithmic error of 0.168 .

\subsection{Mass loss in the $\Gamma_{1}$-Geyser model}

Maeder (1989) discusses the physical considerations of LBV outbursts, the reason for the shell ejections on the timescales involved, which can probably also be applied to the star HR 8752 on its blueward evolution along track 4. We indicated earlier the relevant timescales for HR 8752. Maeder also discusses the response of a LBV to a large shell ejection and a theoretical lightcurve. The light curve from Maeder (1989, Fig. 2) shows the response in $B$-magnitudes to a shell ejection of $1 M_{\odot}$ at various mass-loss rates (mean taken over some ejection time).

For the observations of HR 8752, we make three comments: 1) If our estimate of mass lost (through a shell ejection) is realistic, we find an observed mass ejection of $\approx 0.82 M_{\odot} .2$ ) Although we have only $V$-data available, we can compare our Fig. 13 with Maeder's response to a large shell ejection with a mean massloss over 20 years of $0.05 M_{\odot} / y r$. The general form of the curves seem similar if we think of a long recovery time, but the exact form may be different. 3) The recovery time for the above data 
and mass ejection for a $40 M_{\odot}$ model is 350 years (Maeder 1989 , table on p. 22). The observations indicate a "recovery" time that is significantly longer than the observation time of $\approx 160$ years, and an estimate of some 300-350 years seems reasonable.

\subsection{Conclusions}

If the process that we have observed is a long-term instability cycle that can be described by the $\Gamma_{1}$-Geyser process (Maeder $1989,1992)$, it seems that the severe mass lost as part of the process leads to a much higher efficiency in losing mass than the wind-driven mass-loss does. It also means that for m040e020 the evolutionary time spent within the yellow void I up to $T_{\mathrm{Blue}}=$ 3.90 drops from 6000 to some 1400 years, and for $\mathrm{m} 025 \mathrm{e} 020$ from some 180000 to 1400 years.

This process seems to operate in giant steps of massloss, dividing long evolution times over the yellow void I into a small number of 300-350 year steps, integrating to some 1000-2000 years for part of the evolution in the yellow void I, and possibly also in the yellow void II. The term yellow void may explain the very short time an observable hypergiant stays within its region.

The observations of $V$-magnitude (cf. Fig. 13) seem to indicate the reality of the $\Gamma_{1}$-Geyser process for this fourth step of HR 8752 in yellow void I, shortening evolution time steps of some 2000-10000 years to a small series of mass ejections of each $\approx 300-350$ years process duration.

If the number of steps given by the models is realistic, we hypothesize that the first (large) mass-loss might have occurred 4 cycle times ago, which is a time of some 1200-1400 years.

HR 8752 has reached $T_{\text {Blue }}=3.90$ and the question is whether the star will undergo another cycle, or the star can be expected to leave yellow void I, resume its "regular" evolution, and then later enter yellow void II? To answer this question and verify this prognosis, measurements over a much longer time interval are needed.

\subsection{Historical records}

Since Maeder's (1989) "shell ejection" event seems part of a recurring cycle of long duration and the $V$-magnitude of HR 8752 may have been fainter than $V=6$ (the lower level of naked eye visibility), we search historical astronomical observations for a "new" star observed in the constellation Cassiopeia. HR 8752 has no official constellation assignment but was subsequently designated as V509 Cas at a later date. Would the appearance of a new star due to the intensity brightening as part of the $\Gamma_{1}$-Geyser process have been noticed some $1200-1400$ years ago in the constellation Cassiopeia?

Writing about the new star of 1572, Cyprianus Leovitius (1573) mentioned two possible earlier new stars, approximately in the same region of the sky, appearing in A.D. 945 and 1264. Below we give the medieval latin text followed by a translation. For more details cf. Table A.10.

On the new star: Cyprianus Leovitius: De nova stella (Lavingae, 1573, pp. A2v-A3r):

"Historiae perhibent tempore Ottonis primi Imperatoris similem stellam in eodem fere loco Coeli arsisse Anno Domini 945. Ubi magnae mutationes plurimaque mala, varias Provincias Europae pervaserunt: potissimum propter peregrinas gentes infusas in Germaniam. Verum multo locupletius testimonium in hiftorijs extat de Anno Domini 1264. Quo stella magna $\&$ lucida in parte coeli septentrionali circa sydus Cassiopeae apparuit, carens similiter crinibus, ac destituta motu suo proprio: cum paulo post duae praestantissimae ac florentissimae familiae Germanicae excisae sint: \& inter Electores ac Principes Germaniae summae distractiones fuerint, interregnumque plurimorum annorum cum laniena secutum sit".

\section{Translation:}

"Histories tell us that in the times of Emperor Otto I, in A.D. 945 a similar star appeared in almost the same place of the sky. Various provinces of Europe were affected, generally in a bad way, mostly due to peoples intruding into Germany. We have more testimonies in histories of the year A.D. 1264, when a great and bright star appeared in the northern part of the sky, near the constellation Cassiopeia, also without a tail and a motion of his own: two of Germany's most excellent and flowering families were murdered not long after, there were disagreements between the Electors and Princes of Germany, and there was an interregnum for a long time, followed by a massacre".

The reality of these events are not well-established, though they are not impossible. There were comets in the respective years, but they do not correspond to Leovitius' description (Kronk 1999).

We firstly note that although the two medieval observations above have been advocated as being possible precursors to the appearance in 1572 of Tycho's star (Zsoldos 2010 notes 23, 24), it is interesting that these appearances offer observations that are coincidental in place (Constellation Cassiopeia) and in time (beginning of the shell-ejection cycles) of a possible $\Gamma_{1}$-Geyser process in HR 8752 (and/or $\rho$ Cas).

In addition we conclude this section with the remark that the $\Gamma_{1}$-Geyser process may be the major driving process behind the instabilities in yellow hypergiants and LBVs. It allows stepwise large or giant losses of mass whilst a star is in a region where $\Gamma_{1}$ instabilities can occur in (large parts of) the atmosphere. This mass-loss process is orders of magnitude more efficient than the wind-driven mass-loss process, but is limited to regions where the ionization of $\mathrm{He}$ and $\mathrm{H}$ are extremely important at the same time that the masses of the stars evolving through these regions are insufficient to guarantee stability.

\section{Energy considerations}

Figure 24 gives the "total bolometeric luminosity" of HR 8752 in a linear plot against modified julian day (mJD) with $\mathrm{mJD}=$ JD-2 400000 between 1950 and 1995, where the short lines indicate the luminosity levels with $\mathrm{L} 1=243000$, L2 $=316000$, $\mathrm{L} 3=213000$, and $\mathrm{Lm}=398000$ in solar units of $L_{\odot}=3.85 \times$ $10^{33} \mathrm{erg} / \mathrm{s}$ (Schmidt-Kaler 1982).

Four vertical lines indicate three integration areas of different character, which we name "a", "b", and "c". The vertical lines correspond to $\mathrm{mJD}=43000,43300,45400$, and 48800 , respectively.

\subsection{Excess luminous energy}

To estimate the excess luminous energy freed by the "1973" event, we integrate the luminosity given in Fig. 24 over time in the three indicated sections. The short horizontal lines indicate the luminosity levels. In Table 11, we give the integration between Lm and L3 ("a") with repect to level L2, also for "b", "c", the total of the the three areas, and the mean excess energy loss per day.

Lobel et al. (2003) find for the mass ejection of 2000-2001 for $\rho$ Cas a total energy ejected of $6.1 \times 10^{44} \mathrm{erg}$, over a period 
Table 11. Estimate of "flash" energy and of luminous energy freed, from observations after the 1973 "event".

\begin{tabular}{lcccc}
\hline \hline Block & $\begin{array}{c}\text { Days } \\
\text { (d) }\end{array}$ & $\begin{array}{c}\text { Integral } \\
\text { (Lsun.day) }\end{array}$ & $\begin{array}{c}\text { Energy } \\
\text { (ergs) }\end{array}$ & $\begin{array}{c}\text { Mean energy/d } \\
\text { (ergs/day) }\end{array}$ \\
\hline "e"1 & 1390 & & $7.43 \times 10^{46}$ & $5.35 \times 10^{43}$ \\
\hline "a" & 300 & $4.32 \times 10^{7}$ & $1.437 \times 10^{46}$ & $4.79 \times 10^{43}$ \\
"b" & 2100 & $2.163 \times 10^{8}$ & $7.195 \times 10^{46}$ & $3.43 \times 10^{43}$ \\
"c" & 3400 & $3.621 \times 10^{8}$ & $1.204 \times 10^{47}$ & $3.54 \times 10^{43}$ \\
total $^{2}$ & 5800 & $6.216 \times 10^{8}$ & $2.067 \times 10^{47}$ & $3.564 \times 10^{43}$ \\
\hline
\end{tabular}

Notes. (1) "e" gives the explosive "flash" energy from indirect estimates given in Sect. 6.5 (2) "total" gives the sum of blocks "a", "b", and "c" derived in Sects. 6.1, 6.2, and 6.7, while "e" implicitly includes block "a".

of 100 days, hence a mean ejected energy of $6.1 \times 10^{42} \mathrm{ergs} /$ day. It appears that the HR 8752 " 1973 " event has a total energy loss of at least 300 times that of the 2000-2001 ejection for $\rho$ Cas, and a mean energy loss 5.8 times higher, over a time span that is 60 times longer than $\rho$ Cas. HR 8752 does have smaller "events" that more clearly show the behaviour of the 2000-2001 $\rho$ Cas mass-loss. We refer to the smaller episodes around both 1960 and 1980 that we mention in the main conclusion, end of Sect. 3, and in step 2, Sect. 3.2.

\subsection{Observed timescales of observations of energy emission}

But where is the observed energy stored? We investigate the different characterizations of blocks (a), (b), and (c) in Fig. 24, and identify the differences between the timescales. For block (a), the drop in the luminosity from the beginning of the observations after the 1973 event is quite fast: 300 days for a drop of 56\% of Lm to L2 with respect to L3. This represents a dynamic timescale. On the basis of the earlier parts of this paper, we conjecture that the energy was stored as H-ionization energy in a shell around the central star and liberated through some form of $\Gamma_{1}$-Geyser mechanisms as mentioned in Sect. 4, which possibly carries on into block (b). Block (b) shows the variations in the type 1 (cf. Table 6) but seems to stay on a constant energy level. The timescales are probably also of type dynamic. Block (c) has a completely different character that corresponds to a timescale of type thermal or Kelvin-Helmholtz, similar to that associated with the infall of stellar material onto the central star. We tentatively associate this process with the collapse of an atmosphere from level Lm to L2 along a nearly straight line and try to determine whether the potential energy of an atmosphere that extends half a stellar radius away is responsible for the luminous energy liberated in the process of the atmosphere falling onto the central star. We concentrate however on block (c) and try to identify the sinking of the atmosphere between levels L2 and L3.

With respect to block (c), Lambert et al. (1981) inferred from observations of infrared CO and visual atomic interstellar lines that a shell ejected in 1975 was then falling back onto the star (1979). Lambert \& Luck (1978, p. 419) mention for 1976 “Then, the metallic lines, which are identified with the upper photosphere, were falling down at a relative velocity of $30 \mathrm{~km} \mathrm{~s}^{-1}$ on to the lower photosphere".

In block (a), the energy is freed as internal energy by the deionization of an extended part of the atmosphere. We can only see the last part of this process. In block (b), it is uncertain what is happening, but we expect the process of (a) to continue, while the atmosphere also begins to feel the results of the missing ionization and starts to rain down onto the central star. Is it possible that we see in block (b) the end of the liftoff of ejected material of mass $0.8 M_{\odot}$, which may have been put into a shell over a period of more than 100 years? In block (c), the (fast) energy release of (a) has resulted in an atmosphere that is at least partly de-ionized. The absorption coefficient has changed (rapidly) and there is insufficient luminous energy to support these atmospheric layers. Hence, the layers will fall down onto the central star (central part of the atmosphere). It is in some respects a catastrophe for the atmosphere.

Can we find out how much material is falling down? This would be interesting because it could give an indication of the shell mass contributing to the de-ionized shell zone in block (a) and/ or the shell mass contributing to the luminous energy in block (c). Although we are interested in measurements of $(\Delta M / M)$ (cf. Sects. 5.5, 5.6) for these two zones, we only attempt them for block (c).

\subsection{Determining shell mass}

We assume that the energy for (c) is stored as potential energy in an extended mass around the central star, which is built up over $\approx 300$ years, and that this energy, together with the energy in the ionized region (internal energy), forms the total energy available for a large "event" or explosion (for a part even before block (a)).

We concentrate here on the observations in block (b), and propose the following model. Using the data from Table 9, we take as a model a central star of radius R3 and mass $(M-\Delta M)$ (shell mass) surrounded at $R 2$ by a shell of mass $\Delta M$. The total mass of the system is $M=M 3$.

We consider the potential energy of a mass $\Delta M$ with respect to the central star. This mass is external to the star but falls back when the absorption coefficient is too low for sustainability. This mass adds to the local mass to become the total mass. We write the potential energy as the product of $m \times g \times h$, where $\mathrm{m}$ is the shell mass $=\Delta M, g$ is the acceleration of gravity due to the central star $g=\mathrm{G} M / R^{2}, M=M 3-\Delta M$, and $\mathrm{h}$ is the height of the fall, i.e. the difference in radius of the extended atmosphere with respect to that of the central star $\mathrm{h}=R 2-R 3$.

With the values given above, combining the relevant radius and mass factors in a factor "f" and rewriting for mass in solar units, we have

Potential energy (block (b)) $=m \times g \times h=\Delta M \times(M-\Delta M)$ times the factor "f" with "f" $=9.056 \times 10^{45} \mathrm{erg}$ and total $M=$ $M 3=10.8 M_{\odot}$.

Is the potential energy from this model comparable to the luminous energy of $1.204 \times 10^{47} \mathrm{erg}$ found for block (c)? Equating the potential and the luminous energies and solving for $\Delta M$, we find a value for $\Delta M=1.417 M_{\odot}$ and thus $(\Delta M / M)=0.131$. We find that indeed the potential energy is of the same order as the observed luminous energy, and that a shell mass of $13 \%$ of the total mass is involved. We conclude that the energy may have been stored as a sum of potential, internal, and mechanical energies, but that the potential energy is an essential part of the energy balance.

Only one-half of the potential energy is classically transformed to luminosity. This factor of one-half is a maximum value, which is true in the case of an ideal mono-atomic gas. In this case, we would need a potential energy twice as high as the radiated energy. Solving for the classical case then results in $\Delta M$ (classical) $=3.797 M_{\odot}$ and the mass fraction $(\Delta M / M)($ classical $)=0.358$. 


\subsection{Energy freed by H-recombination}

If the above value of mass falling down onto the central star is attributable to the recombination of hydrogen, we can obtain an estimate of the ("flash") energy released in a process of complete recombination. We find that $1 M_{\odot}$ of ionized hydrogen releases $2 \times 10^{33} \mathrm{~g} \times 6.02 \times 10^{23} / \mathrm{g}($ Avogadro $) \times 13.6 \mathrm{eV} \times$ $1.6 \times 10^{-12} \mathrm{erg} / \mathrm{eV}=2.61 \times 10^{46} \mathrm{erg}$. For the value of shell mass $\Delta M($ classical $)=3.797 M_{\odot}$ and a fraction of $\mathrm{H}$ mass $X=$ 0.75 (solar-type abundance), the shell recombination energy is then $E_{\text {shell }}=7.43 \times 10^{46} \mathrm{erg}$, which is comparable to the energies given in Table 11 .

We conclude that the recombination energy is of the same order and somewhat higher than the energy in block "a" of Table 11 that is integrated between the levels Lm, L2, to L3 over time.

The available recombination energy depends on the partial ionization fraction " $x$ " with $0 \leq$ " $x$ " $\leq 1$. The given values are therefore maximum values.

The estimate for HR 8752 assumes that the gas has temperatures and densities in the layer $\Delta M$ that ensure it becomes (nearly) completely ionized (but for the factor " $x$ ") and subsequently undergoes a (nearly) complete recombination. Are these gas conditions possible in such an extended massive layer?

\subsection{Estimated mean explosive "flash" energy}

There is significant evidence of a dramatic change in HR 8752 going from $\mathrm{mJD}=41560$ (G5 Ia), to $\mathrm{mJD}=41606$ (G5 Ia) (Morgan et al. 1981; Fry \& Aller 1975) and mJD = 41910 (G8-K5) (Luck 1975; Lambert \& Luck 1978; cf also Table A.5) and a measurement on mJD $=42368$ of $(B-V)=1.73$ by the method of McFarlane (Luck 1975; cf. also Table A.7). However, we have no direct measurement of the $V$-magnitude, so that all we can say is that we expect the "flash" to have started at about the date of $\mathrm{mJD}=41910$.

To find the mean explosive "flash" energy, we take the energy of $E_{\text {shell }}=$ " $x " \times 7.43 \times 10^{46}$ erg over a time given by the difference between $\mathrm{mJD}=43300$ (end of "a") and $\mathrm{mJD}=41910$ or $1390 \mathrm{~d}$. Taking " $x$ " $=1$, the maximum mean "flash" energy is then $5.35 \times 10^{43} \mathrm{ergs} /$ day. We add these data separately as block "e" at the beginning of Table 11 for comparison purposes.

\subsection{Discussion}

The energy emitted by HR 8752 during the phases considered here have been released over some three and sixteen years, but we can only make an indirect estimate, given that the direct information misses the largest part of the presumed explosion itself. They show respectively a somewhat faster and a "slower" release, but they combine to produce a high total value.

The measurement of the energy in Sects. 6.1 and 6.2 is based on the evaluation of the luminosity, hence radiated energy. We discuss the use of potential energy that is converted to luminous energy, and then measure the emitted light.

The factor $(\Delta M / M)$ refers to the depth (mass fraction) of the atmosphere to which the $\Gamma_{1}$ de-ionization flash may possibly reach at the beginning of the eruption on the timescale given by $t_{\mathrm{dyn}}$ or $t_{\mathrm{KH}}$ as described in Sect. 5.5 of the paper. A value of $0.1-0.2$ (of the total mass) indicates the mass fraction of the atmosphere undergoing the (Geyser?) instability. It is not a factor of actual mass-loss given in De Jager et al. (1988).

We consider these high energies, if real, as possible indications of later larger explosions of huge energies.

\subsection{Comparing $t_{\text {therm }}$ with observations}

In Sect. 5.5 (cf. Table 9), we gave the timescale values for timepoints L1, Lm, and L3. For a fractional mass $(\Delta M / M)$, we multiply below these timescales with that fraction to find the corresponding timescale, and compare the observed and theoretical timescales.

Using the "1990" timescale of point L3, we find 55.6 years, thus for $(\Delta M / M)=0.131$ a value of $t_{\text {therm }}=7.30$ years (or $2664 \mathrm{~d}$ ), which we compare with the observation of $3400 \mathrm{~d}$ for block (c), and for $(\Delta M / M)=0.358$ a value of $t_{\text {therm }}=$ 19.92 years (or $7274 \mathrm{~d}$ ) again compared with the same observation of $3400 \mathrm{~d}$ for $(\mathrm{c})$. The timescales for $(\Delta M / M)=0.131$ is near to the observed value, which for the classical case leads to a value that is twice as high.

\subsection{Conclusion}

We find that the concept of energy stored as internal and potential energy seems to give results that are consistent with observations and that the shell mass fraction of $(\Delta M / M)$ might be somewhere between 0.131 and 0.358 if the potential energy is the only form of energy in block (c). This validates Maeder's (1989, 1991) Geyser model, although with the addition of storing potential energy in an orbit around the star. It seems that there are two types of energy release involved in the Geyser process, a fast and a slower one. The explosion can probably incorporate both of these energies. Does it take more than 100 years to produce a new large explosion?

If the observations and the treatment to come to the luminosity numbers given in this paper for HR 8752 are correct (cf. also the comments at the end of Sect. 5.2), the total energy of $2 \times 10^{47}$ erg is $\approx 1 \%$ of the energy for SN type II with a total radiative energy of $2 \times 10^{49} \mathrm{erg}$ (UBV colours) and total kinetic energy $1 \times 10^{50} \mathrm{erg}$ (UBV colours) (cf. Duerbeck \& Seitter 1982, Sect. 5.1 p. 234). The SN II have much higher temperatures, but also mass ejections forming supernova ejections of 1.5-2.0 $M_{\odot}$ (or more, loc. cit.). Do the very high energies found here for HR 8752 imply that the star might be very near to catastrophic behaviour?

\section{Conclusions}

The main conclusion of our study is that the instability region that we originally called the yellow evolutionary void, actually consists of two separate instability regions, one for $3.8<$ $\log T_{\text {eff }}<3.95$ and another at higher temperatures $4.05<$ $\log T_{\text {eff }}<4.15$. We have identified four separate timescales in the observations, which are descibed in Table 6. Detailed temperature data are presented from spectral, historical MK, $V$, and $B-V$ observations over a longer time interval. A summary is given in Fig. 10. Detailed physical parameters $T_{\text {eff }}, L$, $R$, and $M$ have been found for the period 1942-1996, together with estimates of the interstellar absorption parameters $E(B-V)$ and $R$-ratio. We have estimated the mass lost in the " 1973 " event as the difference of mass before and after the " 1973 " event. By extending the range of V-observations back to 1840 , it seems that the process time for the indicated changing situation is longer than the time of the record, of more than 150 years. This might be indicative of a recurrent (cyclic) character similar to that proposed for the "Geyser model" (Maeder 1989, 1991) and the one that we have found in the evolutionary models mass lost in consecutive steps. The 1840-1900 data indicate that during part of the cycle the star would be invisible for naked eye 
observation and become visible for only a while. Energy considerations based on the observed (excess) luminous energy data may help us to identify and place constraints on the mechanism making the outbursts and instabilities.

\subsection{Additional comments}

The scenario above is probably not limited to yellow hypergiants. It may also be at least partly applicable to luminous blue variables. An example given by Maeder (1989, 1992 p. 142) for a luminous blue variable of $60 M_{\odot}$ star in the yellow supergiant phase, actual mass $46 M_{\odot}$, describes an ejection of a shell of mass $1 M_{\odot}$, and (in the model) two stable temperatures of $\log T_{\text {eff }}=3.82$ and $\log T_{\text {eff }}=4.10$. The first temperature is consistent with yellow void I. The second temperature is not consistent with temperatures of yellow void I but of yellow void II. Here we would expect the $\Gamma_{1}$-mechanism to operate with helium-ionization, or recombination in the extended atmospheres.

We hypothesize that the main distinction between the two temperatures is that the yellow hypergiants operate with their evolutionary temperatures within the yellow void I, and luminous blue variables in both yellow voids I and II.

Acknowledgements. We are grateful to Drs. L. Mantegazza and S. Parsons for providing information about their observations, to Dr. Hans Schrijver for providing the Tycho measurements and accuracies, to Dr. Peter Jonker for indicating the newly revised HIPPARCOS distance measurement of HR 8752, to Centre de de Données de Strasbourg (CDS) for their data service, to Dr. François Ochsenbein of CDS for his help with archiving the external data at CDS, and to SAO/NASA for their Astrophysics Data System for the availabilty of so many research papers. We thank Dr. Richard Stothers for stimulating and fruitful discussions on evolution considerations for finding blue loops for yellow hypergiants on their redward supergiant evolution. We would like to thank the referee for sharply observing the great importance of studies to understand the nature of unstable atmospheric hydrodynamic phenomena that are still puzzling. I.K. would like to thank his colleagues at Tartu Observatory for their help in getting the spectroscopic time series on HR 8752 from 2000 onward. A.L. acknowledges partial funding from the ESA/Belgian Federal Science Policy in the framework of the PRODEX programme (C90290).

\section{References}

Adams, W. S., \& Joy, A. H. 1919, PASP, 31, 184

Adams, W. S., \& Joy, A. H. 1922, ApJ, 56, 242

Adams, W. S., Joy, A. H., Strömberg, G., \& Burwell, C. G. 1921, ApJ, 53, 13

Adams, W. S., Joy, A. H., Humason, M. L., \& Brayton, A. M. 1935, ApJ, 81, 187

Arellano Ferro, A. 1985, MNRAS, 216, 517

Böhm-Vitense, E. 1981, ARA\&A, 19, 295

Buser, R. 1978, A\&A, 62, 411

Cannon, A. J., \& Pickering, E. C. 1924, AnHar, 99, C

Carnahan, B., Luther, H. A., \& Wilkes, J. O. 1969, Applied Numerical Methods (New York: John Wiley And Sons)

Clenshaw, C. W. 1970, 16, Chapter 3, The polynomial and rational approximation of a function of one variable in Numerical Approximation to Functions and Data, based on a conference organized by the Institute of Mathematics and its Applications, Canterbury, England, 1968, ed. J. G. Hayes (New York: The Athlone Press, University of London, London and Oxford University Press), Book number 485111098

Cleveland, W. S. 1979, J. Am. Statist. Assoc., 68, 361

Cleveland, W. S. 1985, The Elements of Graphing Data (Monterey, California, USA: Wadsworth Advanced Books and Software)

Cleveland, W. S., \& Devlin, S. J. 1988, J. Am. Statist. Assoc., 83, 596

Crawford, D. L., \& Mandwewala, N. 1976, PASP, 88, 917

De Jager, C. 1980, The Brightest Stars (Dordrecht, Holland: D. Reidel Publishing Co.)

De Jager, C. 1984, A\&A, 138, 246

De Jager, C. 1998, A\&AR, 8, 145

De Jager, C., \& Nieuwenhuijzen, H. 1995, 459, 32nd Liege Astrophysical Coll., eds. A. Noels, D. Fraipont Caro, M. Gabriel, N. Grevesse, \& P. Demarque, Université de Liège, Institut d'Astrophysique, 4000 Liege, Belgium
De Jager, C., \& Nieuwenhuijzen, H. 1987, A\&A, 177, 217

De Jager, C., Nieuwenhuijzen, H., \& van der Hucht, K. A. 1988, A\&ASS, 72, 244, 131

De Jager, C., Lobel, A., Nieuwenhuijzen, H., \& Stothers, R. 2001, MNRAS, 327, 452

Duerbeck, H. W., \& Seitter, W. C. 1982, A\&A, 2, 234

ESA 1997, The HIPPARCos and Tycho Catalogues, ESA SP-1200 (Noordwijk, The Netherlands: SP-1200, ESA Publication Division, c/o ESTEC)

Eggen, O. J. 1968, ROB No. 137

Fehrenbach C. 1958, Hdbuch der Physik LI, ed. S. Flügge (Berlin: Springer Verlag), 1

Fernie, J. D. 1967, AJ, 72, 442

Fernie, J. D. 1972, AJ, 77, 150

Fitzpatrick, E. L., \& Massa, D. 2007, ApJ, 663, 320

Flower, P. J. 1977, A\&A, 54, 31

Fry, M. A., \& Aller, L. H. 1975, ApJS, 275, 29, 55

Gorlova, N., Lobel, A., Burgasser, A. J. et al. 2006, ApJ, 651, 1130

Gray, D. F. 1992, The observation and analysis of stellar photospheres, 2nd edn. (Cambridge: Cambridge University Press)

Griffin, R. F., \& Redman, R. O. 1960, MNRAS, 120, 287

Gutiérrez-Moreno, A., \& Moreno, H. 1975, PASP, 87, 425

Gyldenkerne, K. 1955, ApJ, 121, 38

Gyldenkerne, K. 1958, Anap, 21, 26

Hagen, W., Humphreys, R. M., \& Stencel, R. E. 1981, PASP, 93, 567

Halbedel, E. M. 1985, IBVS, 2718

Halbedel, E. M. 1986, IBVS, 2876

Halbedel, E. M. 1988, IBVS, 3170

Halbedel, E. M. 1991, IBVS, 3600

Halbedel, E. M. 1993, IBVS, 3849

Harmer, D. L., Lawson, P. A., \& Stickland, D. J., 1978, Obs, 98, 250

Hayes, J. G. 1970, 84 (Chapter 7 Fitting data in more than one variable), in Numerical Approximation to Functions and Data, based on a conference organized by the Institute of Mathematics and its Applications, Canterbury, England, 1968, ed. J. G. Hayes (New York: The Athlone Press, University of London, London and Oxford University Press), Book number 485111098

Henry Draper Catalogue (AnHar, Vols 91-99) (Cannon and Pickering 1918-1924)

Higgs, L. A., Feldman, P. A., \& Smolinski, J. 1978, ApJ, 220, L109

HIPPARCos: http://cdsweb.u-strasbourg.fr/hipparcos.html and ESA publications

HIPPARCos and Tycho Catalogues, The 1997, ESA SP-1200 (Noordwijk, The Netherlands: ESA Publication Division, c/o ESTEC)

Humphreys, R. M. 1978, ApJS, 38, 309

Humphreys, R. M., \& Davidson, K. 1979, AJ, 232, 409

Iriarte, B., Johnson, H. L., Mitchell, R. I., \& Wisniewski, W. K. 1965, Sky and Telescope, 30, 21

Israelian, G., Lobel, A., \& Schmidt, M. R. 1999, ApJ, 523, L145

Johnson, H. L. 1955, AnAp, 18, 292

Johnson, H. L. 1965, ApJ, 141, 923

Johnson, H. L. 1966, ARA\&A, 4, 193

Johnson, H. L. 1968, ch. 5, Interstellar Extinction, in Stars and Stellar Systems, Vol. VII, "Nebulae and Interstellar Matter", eds. B. M. Middlehurst, \& L. H. Aller (Chicago: The University of Chicago Press)

Johnson, H. L., \& Morgan, W. W. 1953, ApJ, 117, 313

Johnson, H. L., \& Morgan, W. W. 1955, ApJ, 122, 429

Johnson, H. L., Mitchell, R. L., Iriarte, B., \& Wisniewski, W. Z. 1966, Comm. Lunar Planet Lab., 4, 99

Keenan, P. C. 1971, Contrib. Kitt Peak Nat. Obs., 554, 35

Keenan, P. C., \& Pitts, R. E. 1980, ApJS, 42, 541

Kippenhahn, R., \& Weigert, A. 1990, 1994, Stellar Structure and Evolution, Astronomy and Astrophysics Library, 2nd edn. (Berlin: Springer-Verlag)

Kraft, R. P., \& Hiltner, W. A. 1961, ApJ, 134, 850

Kron, E. G. 1958, PASP, 70, 561

Kronk, G. W. 1999, Cometography. A Catalogue of Comets, (Cambridge: Cambridge Univ. Press), 1, 155

Kurucz, R. L. 1979, ApJS, 40, 1

Kurucz, R. L., \& Bell, B. 1995, Atomic Line Data, Kurucz CD-ROM No. 23, Smithsonian Astrophysical Laboratory, Cambridge, Mass., USA

Kurucz, R. L., \& Peytreman, E. 1975, A table of semi-empirical gf values, Special Report 362, Smithsonian Astrophys. Obs., Cambridge, Mass. USA

Lambert, D. L., \& Luck, R. E. 1978, MNRAS, 184, 405

Lambert, D. L., Hinkle, K. H., \& Hall, D. N. B. 1981, ApJ, 248, 648

Lamla, E. 1965, in Landolt-Börnstein, New Series, Volume 6/1, ed. H. H. Voigt (Berlin: Springer-Verlag)

Ledoux, P. 1945, ApJ, 102, 143

Ledoux, P. 1958, Stellar Stability in Handbuch der Physik, ed. S. Flugge (Berlin: Springer-Verlag), 51, 605 
Ledoux, P. 1965, Stars and Stellar Systems, Compendium of Astronomy (Chicago 60637: The University of Chicago Press), 8, 499

Ledoux, P., \& Walraven, Th. 1958, Handbuch der Physik, ed. S. Flügge (Berlin: Springer-Verlag), 51, 353

Lee, T. A. 1970, ApJ, 162, 217

Leovitius, Cyprianus 1573, De nova stella, Lavingae, 1573, pp.A2v-A3r

Ljunggren, B., \& Oja, T. 1965, ARA\&A, 4, 137

Lobel, A. 2001, ApJ, 558, 780

Lobel, A., Achmad, L., De Jager, C., \& Nieuwenhuijzen, H. 1992, A\&A, 264, 147

Lobel, A., Dupree, A. K., Stefanik, R. P. et al. 2003, ApJ, 583, 923

Luck, R. E. 1975, ApJ, 202, 743

MacFarlane, M. J. 1969, PASP, 81, 46

Maeder, A. 1989, in Physics of Luminous Blue Variables, ed. K. Davidson et al. (Dordrecht: Kluwer Acad. Press), IAU Coll., 113, 15

Maeder, A. 1992, in Instabilities in evolved super- and hypergiants, Royal Netherlands Academy of Arts and Sciences, Proc. Internat. Coll. Amsterdam, 26 Febr. - 1 March, ed. De Jager et al., North Holland, Amsterdam, 137

Maeder, A. 2009, Physics, Formation and Evolution of Rotating Stars (Berlin: Springer Verlag)

Maeder, A., \& Meynet, G. 1987, A\&A, 182, 243

Maeder, A., \& Meynet, G. 1988, A\&AS, 76, 411

Mantegazza, L. 1988, IBVS, 3269

Mantegazza, L. 1991, A\&ASS, 88, 255

Mantegazza, L. 1992, A\&A, 265, 527

Mantegazza, L., Poretti, E., \& Antonello, E. 1988, IBVS, 3137

Martin, G. A., Führ, J. R., \& Wiese, W. L. 1988, J. Phys. Chem. Ref. Data, 17, Suppl. nrs. 3 and 4

Meynet, G., Maeder, A., Schaller, G., Schaerer, D., \& Charbonel, C. 1994, A\&AS, 103, 97

Moffett, T. J., \& Barnes, T. G. 1979, IBVS, 1533

Moore, C. E., Minnaert, M. G. J., \& Houtgast, J. 1966, The Solar Spectrum 2935 A to 8770 A, Second revision of Rowland's Preliminary Table of Solar Wavelengths, National Bureau of Standards Monograph 61

Morgan, W. W., \& Keenan, P. C. 1973, ARA\&A, 11, 29

Morgan, W. W., Keenan, P. C., \& Kellman, E. 1943, An Atlas of stellar spectra as mentioned by Nassau \& van Albada (1947)

Morgan, W. W., Keenan, P. C., Abt, H. A., \& Tapscott, J. W. 1981, ApJ, 243, 894

Mueller, G., \& Kempf, P. 1903, Potsdam Publ., Band 14

Mueller, G., \& Kempf, P. 1907, Potsdam Publ., Band 17

Nassau, J. J., \& Morgan, W. W. 1951, ApJ, 113, 141

Nassau, J. J., \& Morgan, W. W. 1952, ApJ, 115, 475

Nassau, J. J., \& Seyfert, C. K. 1946, ApJ, 103, 117

Nassau, J. J., \& van Albada, G. B. 1947, ApJ, 106, 20

Nieuwenhuijzen, H., \& De Jager, C. 1990, A\&A, 231, 134

Nieuwenhuijzen, H., \& De Jager, C. 1995, A\&A, 302, 811

Nieuwenhuijzen, H., \& De Jager, C. 2000, A\&A, 353, 163

Nieuwenhuijzen, H., De Jager, C., \& Cuntz, M. 1994, A\&A, 285, 595

Novotny, E. 1973, Introduction to stellar atmospheres and interiors (New York, London, Toronto: Oxford University Press)

Oja, T. 1963, ArA, 3, 273

Olson, B. I. 1975, PASP, 87, 349

Osthoff, H. 1916, Specola Astronomica Vaticana, Volume VIII
Parsons, S. B., \& Montemayor, T. J. 1982, ApJS, 49, 175

Percy, J. R., \& Zsoldos, E. 1992, A\&A, 263, 123

Piters, A., De Jager, C., \& Nieuwenhuijzen, H. 1988, A\&A, 196, 115

Press, W. H., Teukolsky, S. A., Vetterling, W. T., \& Flannery, B. P. 1992, Numerical Recipes in FORTRAN, 2nd edn. (Cambridge: Cambridge University Press)

Sandage, A., \& Smith, L. L. 1963, ApJ, 137, 1057

Schmidt, E. G. 1972, ApJ, 174, 605

Schmidt-Kaler, Th. 1961a, AN, 286,113

Schmidt-Kaler, Th. 1961b, ZA, 53, 1

Schmidt-Kaler, Th. 1965, in Landolt-Börnstein, New Series, Volume 6/1, ed. H. H. Voigt (Berlin: Springer-Verlag)

Schmidt-Kaler, Th. 1982, in Landolt-Börnstein, Neue Serie, Group 6, Astronomy and Astrophysics, External Supplement to Vol. 1, Subvolume b, eds. K. Schaifers and H. H. Voigt, Springer Verlag, Berlin, also in Lang, K. R. 1992, Astrophysical Data, Planets and Stars, section 9.7 Stellar Temperature, Bolometric Correction and Absolute Luminosity (New York: Springer Verlag), 2, 137

Schuster, M. T., Humphreys, R. M., Smith, N., Davidson, K., \& Gehrz, R. D. 2003, Proc. IAU Symp. 212, ed. K. A. van der Hucht et al., 228

Seares, F. H., \& Joyner, M. C. 1943, ApJ, 98, 244

Smolinski, J., Feldman, P. A., \& Higgs, L. A. 1977, A\&A, 60, 277

Smolinski, J., Climenhaga, J. L., \& Fletcher, J. M. 1989, Physics of Luminous Blue Variables, ASSL, ed. K. Davidson, 157, 131

Stebbins, J., \& Whitford, A. E. 1945, ApJ, 102, 318

Stickland, D. J., \& Harmer, D. L. 1978, A\&A, 70, L53

Stothers, R. B. 1972, PASP, 84, 373

Stothers, R. B., \& Chin, C.-W. 1995, ApJ, 451, L61

Stothers, R. B., \& Chin, C.-W. 2001, ApJ, 560, 934

Strömgren, B., \& Gyldenkerne, K. 1955, ApJ, 121, 43

Unsold, A. 1955, Physik der Sternatmospharen, Zweite Auflage (Berlin: Springer-Verlag)

Van Leeuwen, F. 2007, HIPPARcos, the New Reduction of the Raw Data (Berlin: Springer-Verlag)

Van Leeuwen, F., van Genderen, A. M., \& Zegelaar, I. 1998, A\&AS, 128, 117

Vetterling, W. T., Teukolsky, S. A., Press, W. H., \& Flannery, B. P. 1992, Numerical Recipes Example Book (FORTRAN), 2nd edn. (Cambridge, New York, Oakleigh: Cambridge University Press)

Vyssotsky, A. N. 1941, ApJ, 93, 425

Walker, E. N. 1983, MNRAS, 203, 403

Wiese, W. L., Smith, M. W., \& Miles, B. M. 1969, Atomic Transition probabilities - A critical data compilation, \# NSRDS-NBS. 22

Wilsing, J. 1919, Potsdam Publ., Band 24, No. 74

Yoon, S.-C., \& Cantiello, M. 2010, ApJ, 717, L62

Zsoldos, E. 1986a, The Observatory, 106, 156

Zsoldos, E. 1986b, IBVS, 2913

Zsoldos, E. 1986c, in Luminous Stars and Associations in Galaxies, eds. C. W. H. de Loore, A. J. Willis, \& P. Laskarides, IAU Symp., 116 (Dordrecht: Reidel Publ. Co.), 87

Zsoldos, E. 2010, JHA, 41, 105

Zsoldos, E., \& Oláh, K. 1985, IBVS, 2715

Zsoldos, E., \& Percy, J. R. 1991, A\&A, 246, 441

Zug, R. S. 1931, ApJ, 73, 26 


\section{Appendix A: Introduction to the figures}

Associated with the tables referred to as Table Axx, resp. Bxx, that are available at the sites mentioned in Sect. 1 of the main paper, we give the three figures mentioned in Tables A.9 and B.2.

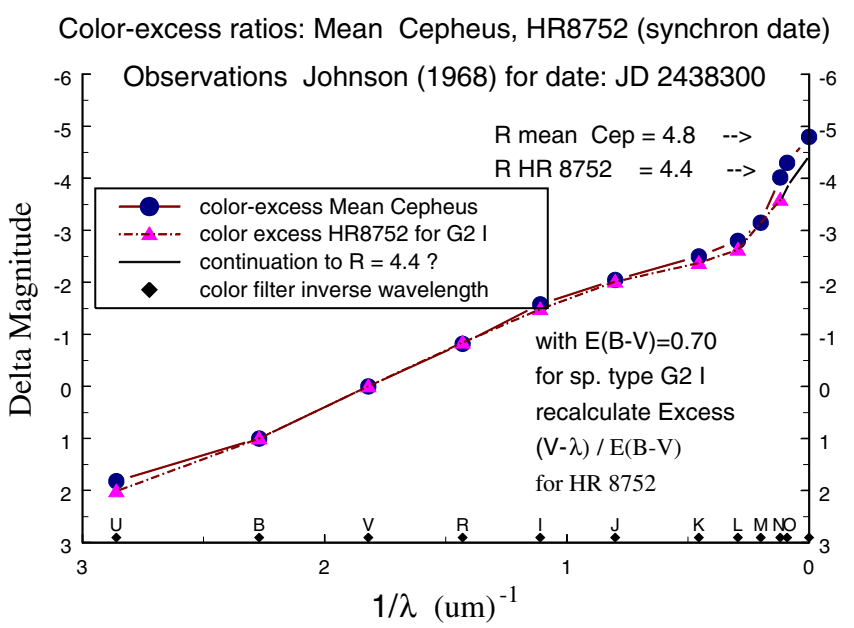

Fig. A.1. The color-excess ratio of HR 8752, recalculated for synchronicity of MK-type, is given as function of $1 / \lambda$, with the plot for the mean of 6 stars in Cepheus from Johnson (1968) as comparison, leading to an redetermination of the R-ratio $R$. We estimate for HR 8752 an $R=4.4$ with an accuracy of +-0.2 in analogy with Johnson's determination of the the $R$-value at $1 / \lambda=0$ for the "mean". With $E(B-V)=0.70$ the absorption in the $V$ color is $A v=3.08$.

Figure A.1 gives the colour-excess ratio of HR 8752, recalculated for synchronicity of MK-type as a function of $1 / \lambda$, after Johnson (1968), together with the mean for 6 stars in Cepheus (from Johnson 1968) as comparison. The data is given in Table A.9, where the above figure is mentioned as Fig. A.9.1.

Figure A.2 gives the temperature calibration for hypergiants, using observational data ( $T_{\text {eff }}$ from De Jager 2001), as a function of s-parameter (De Jager \& Nieuwenhuijzen 1987).

Figure A.3 compares the calibration with the two spectral class relations for Ia and $\mathrm{Ia}+$. The data is associated with Table B.2, where the above figures are mentioned as Figs. B.2.1 and B.2.2, respectively. In De Jager \& Nieuwenhuijzen (1987), the spectral class Ia+ is represented by $b$-parameter $b=0.0$, class Ia by $b$-parameter $b=0.6$.

The calibration line coincides with the Ia and Ia+ lines at high temperature (low value of $\mathrm{s}$ ), then after $s=2$, it stays close
Teff as function of s-parameter (De Jager et al., 1989)

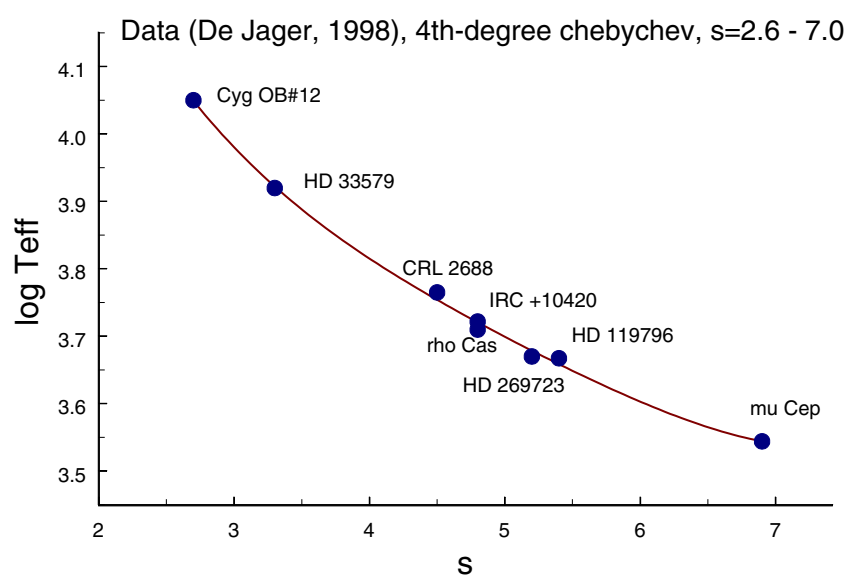

Fig. A.2. The continuous line gives the calibration of hypergiant temperatures based on observational data ( $T_{\text {eff }}$ from De Jager 2001), as a function of $s$-parameter (De Jager \& Nieuwenhuijzen 1987).

Comparison of Teff(s) calibration, data De Jager (1998), and

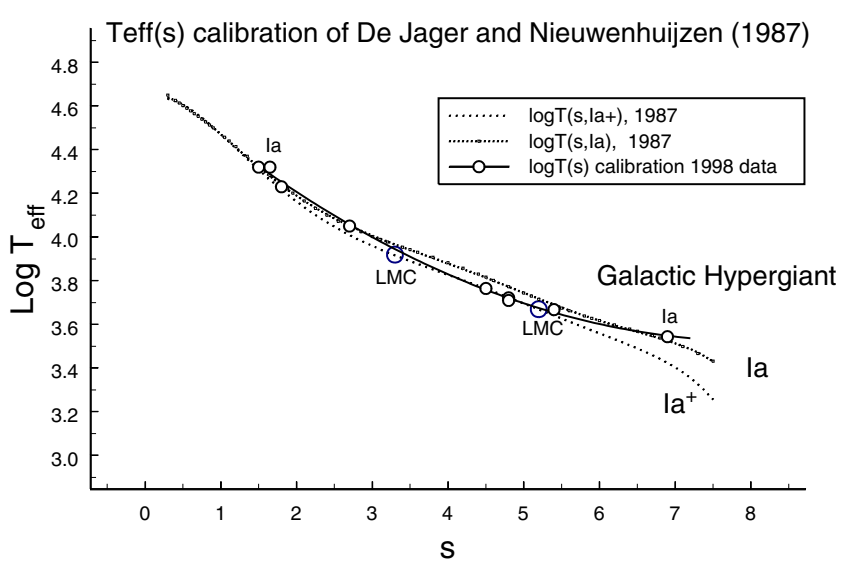

Fig. A.3. The calibration as given in Fig. A.2 is compared with earlier calibration of $T_{\text {eff }}$ as a function of $s$-parameter, and shows it's position between spectral class Ia and Ia+ (De Jager \& Nieuwenhuijzen 1987).

to the Ia line, between $s=3.5-5$, it stays close to Ia+, and then it stays close to the Ia line around $s=6.8$ (with $\mu$ Cep). For practical purposes we limit the fit to the observational s-range of $s=2.6-7.0$. The resulting temperature calibration is valid within the limited spectral range of B8 - M2. 